\title{
Assessing reliability of fatigue indicator parameters for small crack growth via a probabilistic framework
}

\author{
Andrea Rovinelli ${ }^{1}$, Yoann Guilhem ${ }^{2}$, Henry Proudhon ${ }^{3}$, \\ Ricardo A Lebensohn ${ }^{4}$, Wolfgang Ludwig ${ }^{5}$ and \\ Michael D Sangid ${ }^{1}$ \\ ${ }^{1}$ School of Aeronautics and Astronautics, Purdue University, 701 W. Stadium Ave, \\ West Lafayette, IN 47907, United States of America \\ ${ }^{2}$ Laboratoire de Mécanique et Technologie (LMT), ENS Paris-Saclay/CNRS/ \\ Université Paris-Saclay, 61 avenue du Président Wilson, F-94235 Cachan Cedex, \\ France \\ ${ }^{3}$ MINES ParisTech, PSL Research University, MAT - Centre des matériaux, CNRS \\ UMR 7633, BP 87, F-91003 Evry, France \\ ${ }^{4}$ Materials Science and Technology Division, Los Alamos National Laboratory, Los \\ Alamos, NM 87545, United States of America \\ ${ }^{5}$ European Synchrotron Radiation Facility, Grenoble, France, BP 220, F-38043 \\ Grenoble, France \\ E-mail: msangid@purdue.edu
}

\begin{abstract}
Microstructurally small cracks exhibit large variability in their fatigue crack growth rate. It is accepted that the inherent variability in microstructural features is related to the uncertainty in the growth rate. However, due to (i) the lack of cycle-by-cycle experimental data, (ii) the complexity of the short crack growth phenomenon, and (iii) the incomplete physics of constitutive relationships, only empirical damage metrics have been postulated to describe the short crack driving force metric (SCDFM) at the mesoscale level. The identification of the SCDFM of polycrystalline engineering alloys is a critical need, in order to achieve more reliable fatigue life prediction and improve material design. In this work, the first steps in the development of a general probabilistic framework are presented, which uses experimental result as an input, retrieves missing experimental data through crystal plasticity (CP) simulations, and extracts correlations utilizing machine learning and Bayesian networks (BNs). More precisely, experimental results representing cycle-by-cycle data of a short crack growing through a beta-metastable titanium alloy, VST-55531, have been acquired via phase and diffraction contrast tomography. These results serve as
\end{abstract}


an input for FFT-based CP simulations, which provide the micromechanical fields influenced by the presence of the crack, complementing the information available from the experiment. In order to assess the correlation between postulated SCDFM and experimental observations, the data is mined and analyzed utilizing BNs. Results show the ability of the framework to autonomously capture relevant correlations and the equivalence in the prediction capability of different postulated SCDFMs for the high cycle fatigue regime.

Keywords: polycrystalline microstructure, high energy x-ray diffraction microscopy, small crack growth, fatigue indicator parameter, crystal plasticity, machine learning, Bayesian networks

(Some figures may appear in colour only in the online journal)

\section{Introduction}

In engineering, high cycle fatigue (HCF) manifests from cyclic loading with the applied load amplitude well below the material's yield point. Since the lifespan of components subject to HCF exhibit variability, safety factors are utilized during design and inspection schedules are established, in order to guarantee the reliability of the structure. These safeguards directly affect production and operating costs. However, during more than $80 \%$ of a component's lifespan, cracks cannot be detected utilizing common non-destructive evaluation techniques, such as penetrant liquid, simply because cracks are too small $(a<1 \mathrm{~mm})$ [1]. Moreover, only in the last $5 \%-10 \%$ of its lifetime, the crack may be long enough, in order to describe its evolution through phenomenological relationships, such as Paris' Law [2, 3]. Hence, even small improvements in the assessment of material behavior during early stages of crack growth, can improve the overall lifetime predictions of the material/component significantly. Therefore, understanding the small crack (SC) driving force and creating more accurate failure prediction models will help to lower safety factors and the associated production and inspection costs.

According to Forsyth [4], fatigue crack growth is a two stage process: stage I is associated with $\mathrm{SCs}^{6}$, and stage II is associated with long fatigue cracks. Since the introduction of Paris's Law [5], the growth rate of long cracks can be accurately assessed by means of linear elastic fracture mechanics (stage II). For long cracks, the growth rate is monotonically increasing and can be described as a linear trend in a log-log space, and below a certain threshold value (commonly referred as $\Delta K_{\text {th }}$ ), long cracks are not expected to propagate. In contrast, the behavior of SCs is much more complex and rich. For instance, Pearson [6] found that SCs can grow up to 100 times faster than long cracks for the same nominal $\Delta K$ value and more importantly, SC propagation can occur well below $\Delta K_{\mathrm{th}}$. Moreover, SC's behavior can be classified in three different categories [7]:

1. monotonically decreasing growth rate, until SC arrest;

2. a bimodal behavior in which the growth rate is not a state variable: by increasing $\Delta K$, the growth rate first decreases and after reaching a local minimum, increases again until conforming to the long crack's growth rate; and

3. monotonically increasing growth rate, until the SC reaches the transition point and conforms to the long crack's growth rate.

${ }^{6}$ Even though there is a distinction between microstructurally small, small, and short cracks, the term SCs will be utilized to identify microstructurally SC to simplify notation and reading. 
The last, but the more noteworthy feature of SCs, is the vast scatter observed in their growth rate for the same nominal $\Delta K$ value $[8,9]$. In engineering alloys, the variability in the short crack growth rate (SCGR) is strongly influenced by the underlying microstructure and its inherent variability [10].

According to Neumann, stage I propagation is crystallographic in nature and can be idealized as a series of slip processes, occurring on two alternate slip planes [11]. In this case, striations are the result of a combination between work hardening and slip irreversibility. It should be noted that in the Neumann model, ripples observed on the serrated crack surface are not directly related to a single fatigue cycle. McEvily and Bottner [12] studied the correlation between growth rate and grain orientation. They found that orientations leading to high work hardening exhibit higher growth rate, which is itself strongly influenced by the normal stress acting on slip planes at the crack tip. Moreover, they also stated that no simple exponential relationship is able to describe the observed scatter in the crack growth rate, and that cross slip plays a crucial role in the development of (i) the substructures ahead of the crack tip (e.g. dislocations) and (ii) the shape of the crack front. Rice and Thomson [13] investigated dislocation emission from the crack tip. They found a criterion, which if satisfied, allows the material to spontaneously emit dislocations from the crack tip to relax the experienced stress level. They found that for FCC, and some BCC materials, this mechanism is responsible for the crack blunting process and consequently governs the material's ductility.

Unfortunately, due to time/cost constraints and feasibility, experiments cannot elucidate the complete picture of the material behavior. In recent years, with increased computational resources, researchers started using the crystal plasticity (CP) models, originally proposed by Asaro [14]. This technique allows to create a very well controlled environment, even if not completely realistic, in order to understand the impact of specific microstructural features on micromechanical fields. Utilizing $2 / 2.5 \mathrm{D}$ simulations, several authors investigated the role of different aspect of the inherent microstructure variability on the SCGR (i.e. [15-17]).

To achieve a deeper understanding of the physics governing SCGR, many researchers investigated the role of dislocations, by means of molecular dynamics simulations, and correlated the dislocation mechanics to different aspects of the SC behavior. To name a few: Bitzek and Gumbsch [18] investigate the interaction of different kind of preexisting dislocations and the crack front; Sangid et al [19] investigated the role of the grain boundary (GB) character in slip transmission and dislocation nucleation; Argon [20] and Giannattasio and Roberts [21] investigated how dislocation mobility is responsible for crack growth and arrest.

As noted in the aforementioned studies, inherent microstructure variability of engineering alloys is paramount to the scatter observed in the SCGR. While we are able to qualitatively predict the effect of each microstructure feature with a certain confidence, we are still not able to achieve good quantitative predictions when coupling them. Moreover these studies suggest that in order to accurately predict SCGR, there is a need to establish a framework that can account for different microstructural features and micromechanical fields simultaneously, and for their complex interaction [22, 23].

Mughrabi [24] was the first to relate SCGR to the irreversible plastic strain accumulation during cyclic loading. Based on this idea many researchers investigated different non-local damage metrics for crack nucleation, which are commonly referred to as fatigue indicator parameters $^{7}$ (FIPs). Bennet and McDowell [25], based on the work of Fatemi and Socie [26],

7 In this work we will utilize FIP and SCDFM interchangeably, even though the latter should be preferred due to its more self-explanatory meaning. 
proposed a metric based on the irreversibility of the plastic strain and the normal stress applied on the critical slip plane. Moreover, the importance of the opening stress on the critical plane for SC propagation has also been pointed out by Rice [27] and quantified by Tschopp et al through atomistic simulations [28]. Hochhalter et al [29] and Cerrone et al [30] investigated different possible formulations of FIPs. Their findings suggest that different FIP formulations show similar behavior, and hypothesized that FIPs could be used to predict SC propagation. Yeratapally et al [31] investigated the role of different microstructural features, including misorientation and grain size, and the associated micromechanical fields, such as elastic anisotropy and plastic strain accumulation, on SC nucleation life. These finding support the idea that the SCDFM is the result of the micromechanical field variables, and microstructural features acting in concert during the nucleation and propagation stages.

While FIPs have been used to qualitatively compare different microstructure realizations, in order to establish the likelihood of SC nucleation, only a few studies are available for SC propagation. Utilizing the Fatemi-Socie FIP, Castelluccio and McDowell [32] proposed a non-local model for SCGR that is sensitive to the neighboring grains. They assert that this model can qualitatively reproduce SCGR, but also emphasize the need to validate this model against experimental data. Moreover, Musinski and McDowell [33], proposed a model, which is also based on the Fatemi-Socie FIP, to account for the interaction between the crack tip and the closest GB, accounting for their relative distance. The GB character was treated as a second order effect and was discarded. The model was calibrated against one-dimensional experimental data and subsequently applied to a three-dimensional case. They found good agreement between simulation results and the scatter observed for the SCGR.

Inspired by the experimental results obtained from Ludwig et al and Herbig et al [3436], Proudhon and Li [37, 38] introduced a model that utilizes FIPs as a metric to establish the crack propagation direction, and is able to account for both non-uniform crystallographic propagation (e.g. inside a grain, the crack can switch plane of failure) and crack branching. Hence, their model gives the opportunity to reproduce very tortuous and complex SC morphologies. Furthermore, because the geometrical evolution and growth rate of SC are strictly related to the thresholding condition utilized to allow for material failure, they emphasized the need to quantify the failure threshold.

Concluding, recent and past studies emphasize that the actual SCDFM is not related to one physical quantity, but it is the result of complex interactions between material properties, microstructural features, and micromechanical fields. This means that the FIPs, which are simple SCDFMs built upon the anticipated relevant parameters, will not be able to reproduce qualitatively, much less quantitatively, the complex SC growth mechanics. Moreover, due to the lack of cycle-by-cycle experimental data at the relevant length scale and the computational cost associated with simulating a SC propagating through a microstructure in the $\mathrm{HCF}$ regime, none of the SCDFMs have been appropriately validated against experimental data. The goal of this work is to establish the foundations of a probabilistic, data-driven framework, which combines experiments, simulations, and machine learning techniques, in order to discover and quantify relevant correlations between the observed SCGR, micromechanical fields, and microstructural features. The necessary steps required to build the aforementioned framework will be described. Specifically, the experimental high resolution data of a SC propagating into a real microstructure is used as input for CP simulations, in order to compute missing micromechanical fields imposed by the presence of the crack. Experimental and simulations results are combined into one multimodal dataset, from which data will be mined according to a specific procedure. Extracted data will then be used to build probability distributions, which will be exploited by Bayesian networks (BNs), in order to compute the expected residual life (RL) ahead of the crack. To illustrate the capability of the proposed 

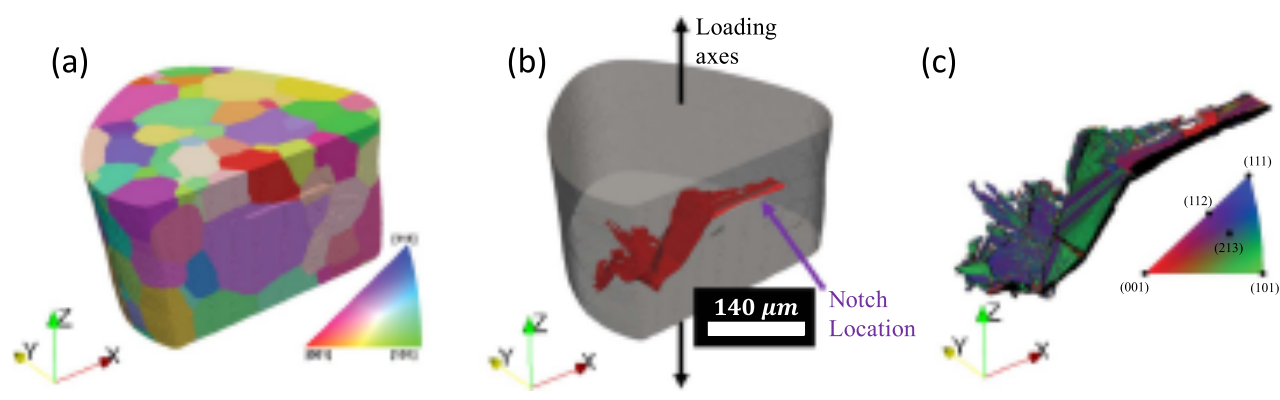

Figure 1. (a) Results of the reconstruction of the initial DCT. Grains are colored according to the IPF color map for cubic symmetry. (b) Schematic of the experiment highlighting: (i) loading axis, (ii) notch position, (iii) the reconstructed crack surface, in red, at the end of the experiment, and (iv) a scale bar. (c) Reconstructed crack's surface; facets of the crack are colored according to their local crystallographic orientation w.r.t. to the IPF color map.

framework, various postulated SCDFMs are selected, and the correlations between their values and the experimentally observed RL are analyzed.

\section{Methods}

\subsection{Experiments}

The dataset utilized in this work is the result of a technique called three-dimensional x-ray tomography of short cracks and microstructure (3DXTSM), which is the combination of diffraction contrast tomography (DCT), multiple phase contrast tomography (PCT) scans, and state-of-the-art reconstruction and segmentation techniques. For more details of the experimental techniques, the reader may refer to [34-36]. It should be noted that DCT allows for three-dimensional grain shape reconstruction and 3DXTSM results are able to describe the geometry of a SC growing into the associated microstructure with high fidelity (micrometer voxel size).

In this study, the material is a near $\beta$-titanium alloy, VST55531. This material is a highstrength titanium forging alloy with minimum ultimate tensile strength of $1240 \mathrm{MPa}$. The $\beta$ transus temperature is $803^{\circ} \mathrm{C}$. The sample studied in this work [35], which has a diameter of $\approx 350 \mu \mathrm{m}$ and a pseudo triangular cross-section, was solution annealed at $843{ }^{\circ} \mathrm{C}$ for $2 \mathrm{~h}$ under vacuum and then air cooled. After the heat treatment, a fully static recrystallization of the $\beta$ phase was obtained with a mean grain diameter of $65 \mu \mathrm{m}$.

A notch (width $\approx 140 \mu \mathrm{m}$, depth $\approx 25 \mu \mathrm{m}$ ) was created utilizing a focused ion beam machining and a single DCT scan (voxel-size $1.4 \mu \mathrm{m}$ ) was performed to obtain the initial microstructure (see figures 1(a), (b)). The sample was subjected to fatigue loading (see table 1 for fatigue loading conditions and figure 1(b) for the orientation of the applied fatigue loading), utilizing the fatigue loading machine developed by Buffière et al [39], which allows for in situ (interrupted) synchrotron scans. Sequentially, every 1000 cycles the fatigue test was interrupted, in order to perform a PCT scan (voxel-size $0.7 \mu \mathrm{m}$ ) of the sample. To avoid crack closure effect and keep the crack open enough to be captured, each PCT scan was performed at maximum load.

The first observation of the crack propagating from the notch was at 34000 cycles, and the experiment was interrupted after the crack surface reached the left edge of the specimen at 
Table 1. Fatigue test loading conditions.

\begin{tabular}{cccc}
\hline $\max \sigma_{33}(\mathrm{MPa})$ & $\min \sigma_{33}(\mathrm{MPa})$ & $R \sigma$ & Frequency $(\mathrm{Hz})$ \\
\hline 320 & 10 & 0.03125 & 25 \\
\hline
\end{tabular}

129500 cycles. Moreover, in order to compensate for the higher growth rate, the frequency at which PCT scans were collected was increased, by reducing the interval between scans from a scan every 1000 cycles to a scan every 500 cycles after the experiment reached 110000 cycles.

Figure 1(c) represents the orientation of crack surface according to its local crystallographic orientation. As can be noted, this image emphasizes the fact that failure occurs not only on the primary planes, $\{110\}$ for BCC materials, but also on secondary and tertiary planes, $\{112\}$ and $\{123\}$, respectively. In order to quantify the relative importance of each plane class and understand the number of slip systems required in the CP simulations, inverse pole density plots were constructed for the fracture surface (figure 2). Each facet of the crack was assigned to the slip plane exhibiting the lowest misorientation angle $\alpha$. Moreover, a threshold of $\alpha_{\mathrm{th}}=15^{\circ}$ has been utilized to distinguished between crystallographic and noncrystallographic failure. The total contribution to failure of each plane class has been computed. Results are summarized in table 2. It should be noted that the value of $\alpha_{\mathrm{th}}=15^{\circ}$ is arbitrary. Moreover, decreasing the value of $\alpha$ would increase the area associated to highly misoriented planes, mainly at the expenses of regions assigned to the $\{112\}$ and $\{123\}$ plane class (with $\alpha_{\mathrm{th}}=10^{\circ}$, the highly misoriented surface is $\approx 26 \%$ of the entire crack surface). As can be noted, failure occurs on all the slip planes, and not only on the primary $\{110\}$ planes [40].

\subsection{Simulations}

3DXTSM does not provide any data about macro/micro-mechanical fields. Efficient computational techniques are a strong requirement to simulate cyclic loading of polycrystalline aggregate with information at the slip-system length scale. To obtain this information at the appropriate length scale, we choose to utilize the elasto-viscoplastic fast Fourier transform solver (EVP-FFT) proposed by Lebensohn et al [41], and based on theory originally proposed by Moulinec and Suquet [42]. Algorithmic details of the EVP-FFT model can be found in [41]. The EVP-FFT model offers significantly improved computational efficiency compared to finite element based CP models, thus it is better suited for cyclic loading. The code utilized in this study is a custom, parallel implementation developed at Purdue University.

Recalling that the material of interest is a near $\beta$-Titanium alloy, the following flow rule and hardening law (equations (1)-(4)) have been selected from literature [43] and modified for the observed cyclic softening behavior (see figure 3 ).

$$
\begin{aligned}
& \dot{\gamma}^{\alpha}=\dot{\gamma}_{0}\left(\frac{\left|\tau^{\alpha}\right|}{g^{\alpha}}\right)^{n} \operatorname{sign}\left(\tau^{\alpha}\right), \\
& \dot{g}^{\alpha}=-\sum_{\beta=1}^{N} H^{\alpha \beta}\left|\dot{\gamma}^{\beta}\right|=-\sum_{\beta=1}^{N} q^{\alpha \beta} h^{\beta}\left|\dot{\gamma}^{\beta}\right|,
\end{aligned}
$$




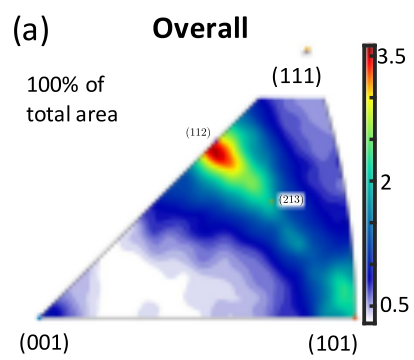

(d) Failure on $\{110\}$

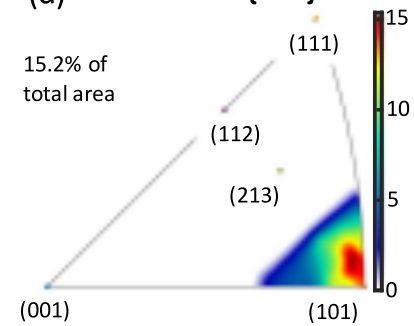

(b) Low misoriented

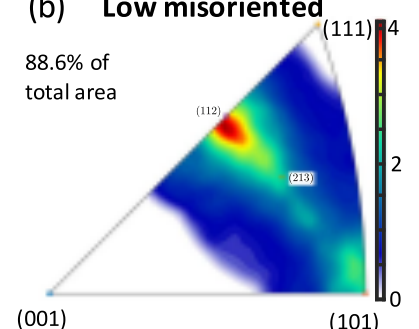

(e) Failure on $\{112\}$

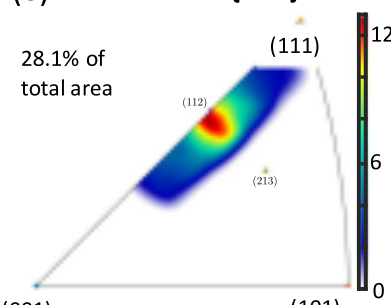

(c) Highly misoriented

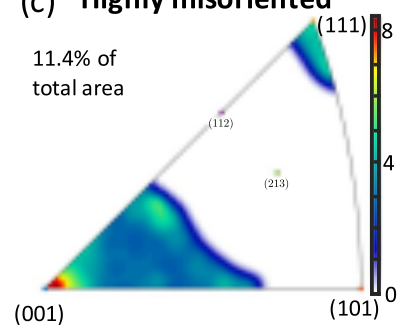

(f) Failure on $\{123\}$

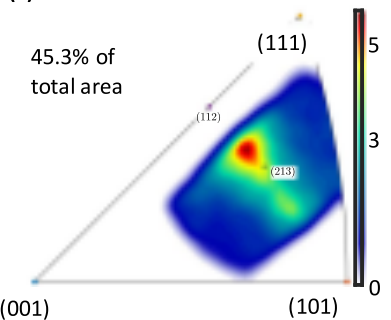

Figure 2. Inverse pole density figures representing the experimentally observed probability of failure of the reconstructed crack surface facets, and the percentage of the crack surface assigned to each subset. (a) Overall distribution; (b) density of the low misoriented facets $\left(\alpha \leqslant 15^{\circ}\right)$; (c) density of the high misoriented facets $\left(\alpha>15^{\circ}\right)$. Subfigures (d)-(f) depict the distributions of low misoriented crack facets further subdivided by the assigned family of slip planes. More specifically: (d) primary slip system $\{110\}$; (e) secondary slip system $\{112\}$ plane; (f) tertiary slip system $\{123\}$ plane. As a note, color scale of each subfigure is normalized against its own subset of data.

Table 2. Percentage, weighted by area, of the crack's facets assigned to each slip plane.

\begin{tabular}{lcc}
\hline & Original crack surface & Voxelization results \\
\hline$\{110\}$ & $15 \%$ & $19 \%$ \\
$\{112\}$ & $28 \%$ & $28 \%$ \\
$\{123\}$ & $45 \%$ & $47 \%$ \\
High misorientation & $11 \%$ & $5 \%$ \\
\hline & \\
$h^{\alpha}=h_{s}^{\alpha}+\operatorname{sech}^{2}\left[\left(\frac{h_{s}^{\alpha}-h_{0}^{\alpha}}{\tau_{s}^{\alpha}-\tau_{0}^{\alpha}}\right) \Gamma\right]\left(h_{s}^{\alpha}-h_{0}^{\alpha}\right)$, \\
$\Gamma=\int_{0}^{T} \sum_{\alpha=1}^{N}\left|\dot{\gamma}^{\alpha}\right| \mathrm{d} t$,
\end{tabular}

where $\alpha, \beta$ are the slip system index, $\tau^{\alpha}$ is the resolved shear stress, $g^{\alpha}$ is the critical resolve shear stress, $\dot{\gamma}^{\alpha}$ is the plastic shear strain rate, $\dot{\gamma}_{0}$ is a scaling constant, $h_{0}^{\alpha}, h_{s}^{\alpha}$, are respectively single slip initial and saturation hardening rate, $h^{\alpha}$ is a variable scaling the effect of slip rate on the update of the critical resolved shear stress, $\Delta \tau^{\alpha}=\tau_{s}^{\alpha}-\tau_{0}^{\alpha}$ is the maximum allowable variation of the initial critical resolved shear stress, $q^{\alpha \beta}$ is a parameter representing self and latent hardening ( $q=1$ if $\alpha=\beta, 1.4$ otherwise), $\Gamma$ is the accumulated total plastic 

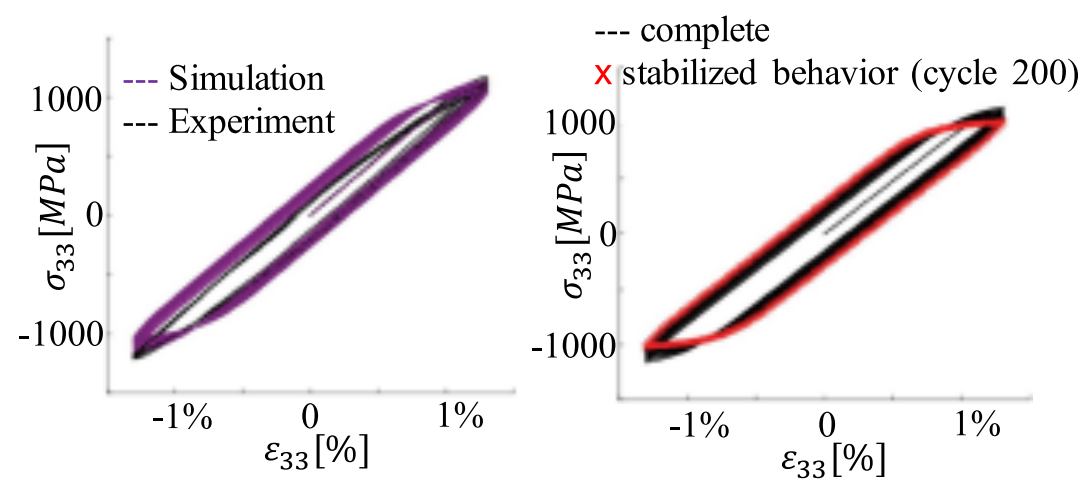

Figure 3. (a) Comparison of the macroscopic stress/strain behavior between experiment (cycle range 1-5) and simulations (cycle range 1-200). (b) Highlight of the stabilized cyclic behavior (cycle 200).

shear at an integration point, and $\dot{g}^{\alpha}$ defines the variation of the critical resolved shear stress. Based on the results of the crack surface analysis, all 48 slip systems for a BCC material are utilized in the simulations, i.e. in equations (2) and (4), $N=48$. The initial critical resolved shear stress $g^{\alpha}$ of primary and secondary slip systems, namely $\{\overline{1} 10\}\langle 111\rangle$ and $\{11 \overline{2}\}\langle 111\rangle$, is the same, while a slightly higher value has been utilized for the tertiary slip system $\{12 \overline{3}\}\langle 111\rangle[44]$.

Experimental data representing the macroscopic behavior of the material were acquired based on a much larger sample than the one utilized for the 3DXTSM experiment. A statistically equivalent microstructure, with more than 300 randomly oriented grains, was generated with Dream3D [45], and utilized to calibrated the EVP-FFT model against cyclic load data (figure 3(a)). Moreover, when dealing with softening materials, some care should be taken to ensure the stability of the solution. To verify that the model would converge to a stable solution and will not suffer from numerical instabilities, 200 cycles were performed on the random periodic microstructure.

Moreover, the authors would like to recall that, due to the unavailability of experimental data at the microscale level, the fitting of the CP parameters have been performed at the macroscale level. Hence, even though results are averagely accurate there may be some local discrepancy between the actual micromechanical fields and the simulated ones. As a consequence, care should be taken in extrapolating the following results and conclusions outside their domain of pertinence. Moreover, in order to validate the following analysis, a comparison between the CP-FFT and CP-FEM simulations for tensile loading with the initial crack geometry has been completed. The results show good agreement of the micromechanical fields in the proximity of the crack and the ability of the CP-FFT solver to correctly replicate their local variations [46]. Results of stabilized behavior are shown in figure 3(b). Elastic constants have been selected from the literature [47] and are reported in table 3 together with the fitted hardening law parameters. It is also worth mentioning that, even though the EVP-FFT solver requires periodic boundary conditions, the resulting distortion of the micromechanical field disappear very quickly. For simulations utilizing the microstructure resulting from the DCT, which is not periodic, the effect of periodicity completely vanishes in less than 10 voxels from the bounding box limit. Care has been taken during data mining in order to avoid sampling from these regions. 
Table 3. Summary of elastic and plastic constants utilized for the simulations.

\begin{tabular}{lllr}
\hline Hardening parameter & & \multicolumn{2}{c}{ Elastic constants } \\
\hline$\dot{\gamma}_{0}\left(\mathrm{~s}^{-1}\right)$ & $2.5 \mathrm{E}-06$ & $\mathrm{C}_{1111}(\mathrm{GPa})$ & 167 \\
$n$ & 10 & $\mathrm{C}_{1212}(\mathrm{GPa})$ & 115 \\
$\tau_{0}^{\alpha}(\mathrm{MPa})$ & 50 & $\mathrm{C}_{2323}(\mathrm{GPa})$ & 44 \\
$\tau_{s}^{\alpha}(\mathrm{MPa})$ & 0 & & \\
$h_{o}^{\alpha}(\mathrm{MPa})$ & 500 & & \\
$h_{s}^{\alpha}(\mathrm{MPa})$ & 0 & & \\
$g_{0}^{\alpha}(\mathrm{MPa})\{110\}$ and $\{112\}$ & 405 & & \\
$g_{0}^{\alpha}(\mathrm{MPa})\{123\}$ & 415 & & \\
\hline
\end{tabular}

The surface mesh (built from triangular elements) representing the experimental crack surface is the result of PCT scans reconstructions and surface post-processing procedures (for further details refer to Herbig et al [35]). PCT scans results are three-dimensional voxelized images, in which each voxel has an intensity value. When thresholding is applied to identify locations belonging to a certain phase, the resulting regions are volumes and not surfaces. The fact that a crack volume, and not a crack surface, is identified from the thresholding, allows for identification of the surface enclosing the cracked volume. This gives the possibility to reconstruct both the upper and lower crack surfaces. In this work, the upper (lower) surface is identified by the mesh elements having a normal with a positive (negative) $z$ value (see figure 1 for the reference system). As a note, having two surfaces is instrumental to completely describe the crystallographic surface orientation of each element in case of transgranular propagation. In other words, if failure occurs at a GB, the crack surface can account for different adjacent grain features, as applicable.

Morphological and temporal information can be assigned to each element of the aforementioned surfaces. In fact, knowing the location of the surface element, it can be assigned to the grain in which it is embedded, allowing the identification of its crystallographic orientation; also, because multiple PCT scans have been recorded, the cycle of failure of each surface element can be determined (with an uncertainty tolerance given by the interval between two subsequent PCT scans).

For a suitable input to the EVP-FFT solver, the crack surface needs to be voxelized. Some care should be taken for an optimal voxelization, mainly for three reasons: (i) the reconstructed crack surface represents a 3D non-simply connected domain (e.g. it has voids), (ii) the crack surface facets' (e.g. elements) size is not uniform, it contains elements which could span more than one voxel and more than one element could be present in each voxel, and (iii) to maintain the sharpness of the crack. In response to these issues: (i), in most cases, voids are artifacts arising due to the limitation of the PCT resolution, subsequent thresholding, and surface smoothing process. A direct voxelization would result in small ligaments of materials carrying the entire load, thereby obscuring the overall simulation results. These small voids need to be identified and filled. To avoid this problem, the voxelization procedure searches for voids and fills them with neighboring information, i.e. voids are filled utilizing failure orientation and cycle number from neighboring surface elements. (ii) A refinement procedure of the crack surface has been developed. The refinement procedure divides large surface elements until their area can be contained inside a single voxel (voxel edge length $1.4 \mu \mathrm{m}$ ), and the ratio between the maximum and the minimum length of each triangle edge is above 0.5 . Moreover, if more than one surface element is present inside a single voxel, the one exhibiting the larger area is utilized as a reference to assign the temporal and 

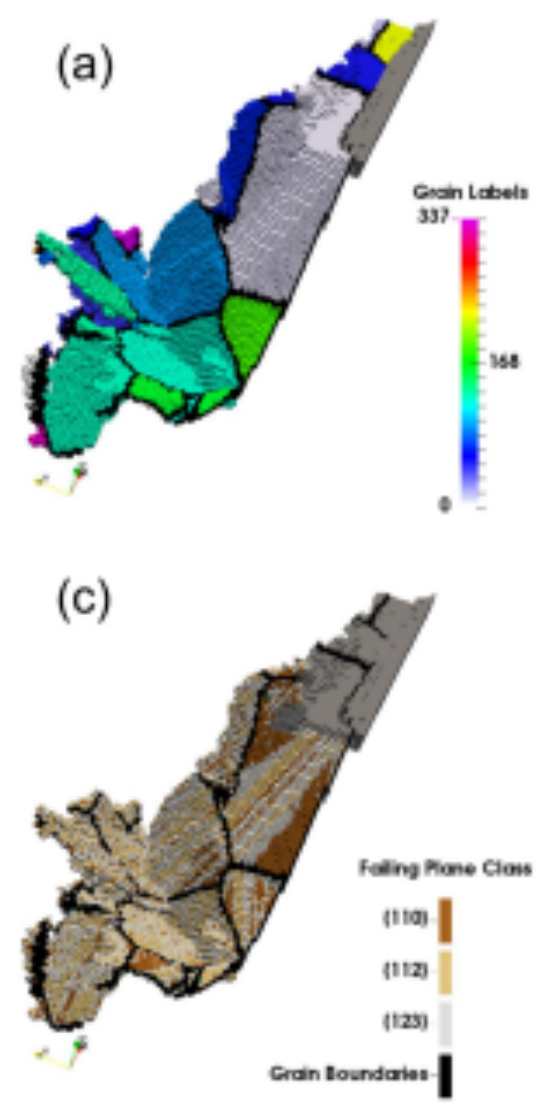

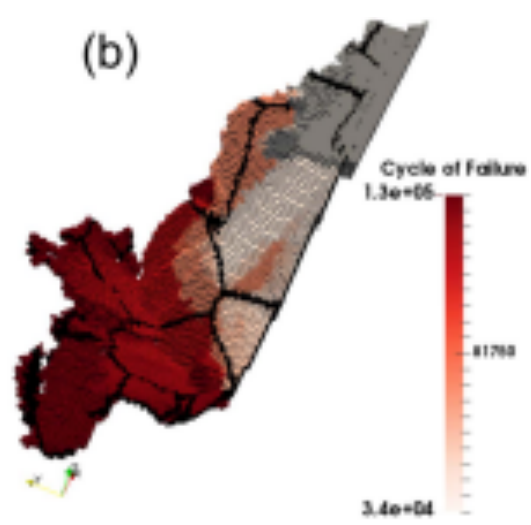

(d)

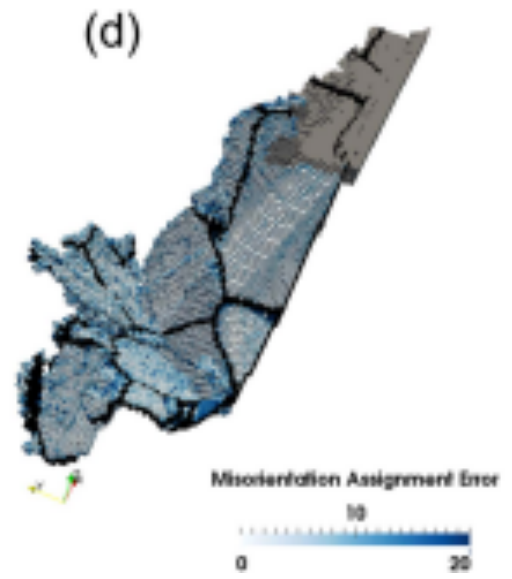

Figure 4. Results of the crack surface voxelization process crack surface colored by (a) grain label; (b) cycle of failure; (c) assigned plane of failure class; (d) misorientation angle between the assigned plane of failure and the crack surface plane of failure.

morphological information to the voxel. (iii) Only the upper surface has been voxelized (this choice is arbitrary). Even if this appears to be a solution that results in a loss of information, in case of transgranular propagation, this step is used to insert the crack as a void phase in the EVP-FFT simulations, but the other surface information is retained for the rest of the analysis procedure. A statistical comparison between slip plane present in the original crack surface and the one assigned during the voxelization procedure is presented in table 2 . Images of the results of the voxelization procedure can be seen in figure 4 .

Furthermore, in order to avoid redundant results and an excessive number of simulations, crack snapshots in which crack growth was deemed insignificant have been combined. A new crack step is defined each time the crack increases its surface by at least $2.5 \%$. This reduced the number of required simulations from 90, which is the original number of snapshots, to 27 . Results of the lumping procedure (gold bars) including a sensitivity analysis along with a full list of the original data are depicted in figure 5. Simulations with the crack geometry ranging from 34000 to 81000 cycles have been performed according to results of the lumping procedure (figure 5). Moreover, we should recall that, because we want to identify the SCDFM, without biasing the BN results, in the simulations the crack is not allowed to grow 
(a)

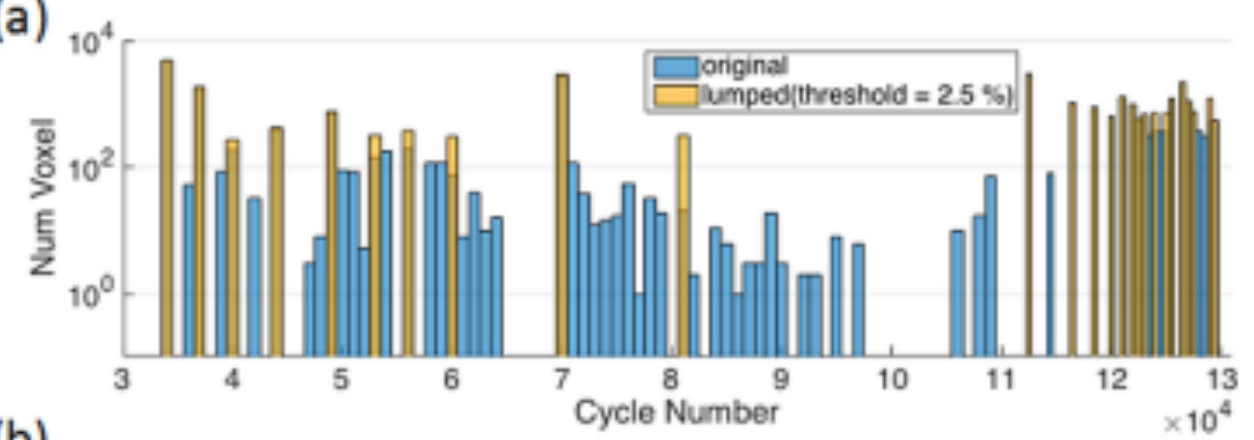

(b)

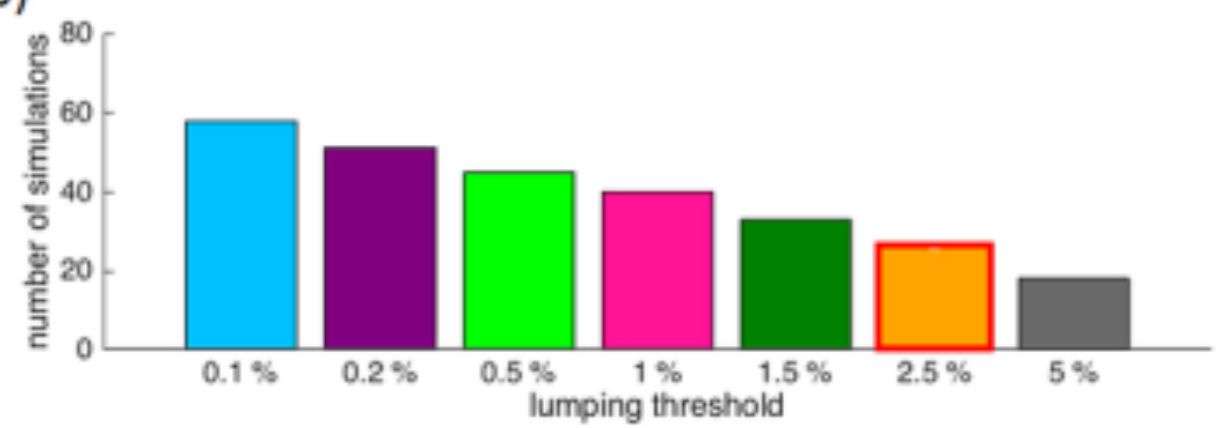

Figure 5. Crack lumping results. (a) Increment of the crack surface in voxels between the current and the previous crack snapshot. (b) Number of simulation required to describe the overall crack evolution as function of the lumping area threshold. The red contour highlights the selected threshold.

(i.e. simulations of various static crack geometries are performed). Moreover, once the voxelization procedure is completed for all the snapshots, the RL of voxels experiencing failure can be computed subtracting the cycle at which failure will be observed and the cycle number associated with the snapshot of interest.

The result of the initial DCT is a grid of $384 \times 384 \times 450$ voxels with a voxel size of $1.4 \mu \mathrm{m}$, of which only the central part $(384 \times 384 \times 192)$ is used in both simulation and analysis. Results of the voxelization procedure are then used as input for the CP simulations, for each new crack snapshot resulting from the lumping procedure. Simulation boundary conditions are imposed, in order to mimic the experimental conditions (i.e. same maximum stress, stress ratio, frequency, etc). For each new crack snapshot, one simulation is performed. The result of the aforementioned simulation will be the following micromechanical fields mapped onto a voxelized (regular) grid:

- Stress tensor $\sigma$

- Elastic strain tensor $\varepsilon_{\mathrm{EL}}$

- Plastic strain tensor $\varepsilon_{\mathrm{PL}}$

- Resolved shear stress for each slip system $\tau^{\alpha}$

- Accumulated plastic shear strain for each slip system $\Gamma^{\alpha}$

Also, to acquire information regarding slip irreversibility and the evolution of the micromechanical fields, 100 cycles are performed for each crack snapshot. Simulation results are exported 3 times per decade (i.e. cycle 1, 2, 5, 10, 20, 50, 100) at maximum load. It 
should also be noted that, in contrast to the finite elements method, due to the meshless nature of the EVP-FFT solver, the material history can be directly translated from one crack snapshot to the next. In this study, the material history is retained between the simulation of the current snapshot to the next. To clarify, no extrapolation is performed between two different snapshot mainly for one reason: the micromechanical fields imposed by the presence of the crack is overwhelming in magnitude compared to the ones imposed by the presence of microstructural features (i.e. grain boundaries). In other words, the amount of plasticity induced in proximity of the crack front by the presence of the crack in few cycles is much higher than the one imposed by micromechanical features.

Once the simulations are complete, the FIPs can be computed through post processing the results of the micro-mechanical fields. For this study, eight different FIP formulations have been selected from the literature (i.e. [10, 48, 49]), and the correlation between their values and the experimentally determined RL are computed and analyzed. The eight different FIPs selected from the literature are described by the following equations:

$$
\begin{aligned}
& D 1=\max _{\alpha}\left|\Gamma^{\alpha}\right|, \\
& D 2=\max _{p} \sum_{\alpha=1}^{N_{s}}\left|\Gamma_{p}^{\alpha}\right|, \\
& D 3=\sum_{\alpha=1}^{N}\left|\Gamma^{\alpha}\right|, \\
& D 5=\max _{p} \sum_{\alpha=1}^{N_{s}}\left|\Gamma_{p}^{\alpha}\right|\left(1+k \frac{\sigma_{n}^{p}}{\sigma_{Y}}\right), \\
& E 1=\max _{\alpha}\left|\tau^{\alpha} \Gamma^{\alpha}\right|, \\
& E 2=\max _{p} \sum_{\alpha=1}^{N_{s}}\left|\tau_{p}^{\alpha} \Gamma_{p}^{\alpha}\right|, \\
& E 3=\sum_{\alpha=1}^{N}\left|\tau^{\alpha} \Gamma^{\alpha}\right|, \\
& E 5=\max _{p} \sum_{\alpha=1}^{N_{s}}\left|\tau_{p}^{\alpha} \Gamma_{p}^{\alpha}\right|\left(1+k \frac{\sigma_{n}^{p}}{\sigma_{Y}}\right),
\end{aligned}
$$

where $\alpha$ represents the slip system number, $N_{S}$ is the number of slip system associated with a specific slip plane, $N$ is the total number of slip system, $p$ is the index representing the slip plane, $k$ is a scaling factor and is set to 0.5 as originally suggest by Fatemi and Socie [26], $\sigma_{n}^{p}$ is the opening stress acting on a specific slip plane, $\langle\cdot\rangle$ are the Macaulay brackets (i.e. $\langle x\rangle=x$ if $x>0,0$ otherwise), and $\sigma_{Y}$ is a scaling constant associated with macroscopic yield stress of the material and is set to $1240 \mathrm{MPa}$. D1 through D5 are computed locally (i.e. at each integration point), and represent: $D 1$ - slip system with maximum accumulated plastic shear strain, D2-slip plane with maximum accumulated plastic shear strain, D3-total accumulated plastic shear strain, D5-slip plane subject to opening stress with maximum accumulated plastic shear. E1 through E5 are the energetic counterparts representing dissipated energy. Note that $D 4$ is equivalent to $E 2$ and is therefore not included in the list. 


\subsection{Identification of the crack's front and data mining}

With the simulation framework established, a data mining procedure is needed to meaningfully use the data. The performed simulations provide an enormous amount of data; while a significantly large volume is required to account for the influence of grain clustering, SCGR is affected by a region directly ahead of the crack tip, in which GBs and complex stress/strain state imposed by microstructural features and macroscopic boundary conditions play a significant role. More precisely, this region is located in the vicinity of the crack front.

The identification of the crack front is a critical step for the data mining procedure, because it will: (i) provide a reference to establish the distance from the crack front, (ii) serve as a base for the identification of the mining volume. Because short cracks are tortuous and exhibit bifurcation behavior, the identification of the crack front is not trivial. For these reasons, the following numerical procedure has been developed and applied to each crack snapshot:

1. Construct the surface mesh of the voxelized crack, utilizing the quick mesh feature of Dream3D [45]. Then, a Laplacian smoothing algorithm is applied to the aforementioned surface mesh to allow for elements belonging to the crack front to slightly reorient. This process produces sharper edges near the crack front and a smoother surface elsewhere.

2. Identify elements belonging to the top and bottom surface (as described previously) and their common nodes, which are the one associated to elements belonging to both the top and bottom surface simultaneously.

3. The linkage of common nodes is embedded in adjacency matrix of the smoothed mesh ${ }^{8}$. Through a Dulmage-Mendelsohn permutation [50], connected paths can be identified and their lengths can be computed. Nodes belonging to long connected paths represent the location of the crack front. On the other hand, short paths are artifacts resulting from step 1 and 2, and therefore thresholding is applied to remove them.

4. The crack's front is extracted, and voxel belonging to the crack front are identified.

Step 3 needs to be performed online (e.g. crosschecked by the user) to guarantee that the threshold value is adequate. Results of this procedure for a specific crack snapshot are depicted in figure 6(a).

Once the location of the crack front is established for each crack step, the Euclidean distance of each integration point from the crack can be computed for every crack step. The mining volume is identified as a solid toroid having the crack front as its centerline and a diameter $D$, which is chosen sufficiently large to include the plastic zone around the crack front. It should be noted that this procedure will also sample behind the crack front, allowing the network to learn from whole spectrum of possibilities, hence reducing the possible bias related to an incomplete dataset. In other words, we want the data to be representative of all possible failing/non-failing behavior, e.g. behind the crack front, no failure should occur. Results will be presented for a mining volume with a radius of $140 \mu \mathrm{m}$, which is slightly greater than twice the average grain diameter. This choice is dictated by the fact that we want to make sure that the network will learn that propagation and failure occurs starting from the main crack surface and not anywhere else in the mining volume (e.g. a new crack is not initiating). Once the mining volume is identified for each crack snapshot, all the voxels with an associated RL are selected (i.e. all the ones that are going to fail in any future crack snapshot). Moreover, in order to understand if FIP values carry enough information to discriminate whether failure is going to happen or not, an equivalent number of non-failing

8 The adjacency matrix is the one whose components characterized the linkage between nodes. 
(a) Crack Front

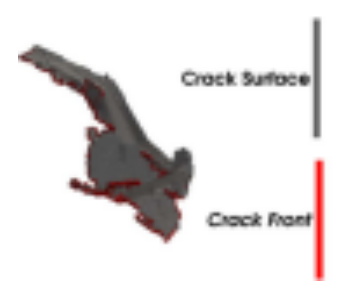

(b) Mining Volume Selection

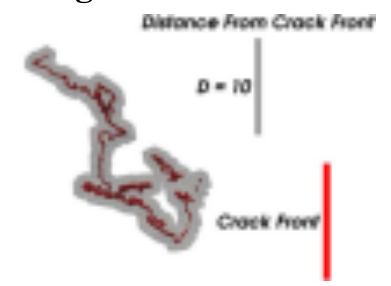

Figure 6. (a) Results of the crack front identification procedure; (b) selected mining volume around the crack front.

points are randomly selected inside the mining volume. Also, in order to alleviate the effect of possible oscillations in the data, once a suitable random point is found, data is collected in small clusters (e.g. all the integration points inside a sphere of radius $2.8 \mu \mathrm{m}$ with center at the randomly selected non-failing points). For each crack snapshot, data are collected 3 times per decade, i.e. after 1, 2, 5, 10, 20, 50, and 100 cycles. Results of the mining procedure is a set of more than 3 million independent observations.

\subsection{Bayesian network}

Due to the nonlinear effects of the interaction between microstructural features, micromechanical fields, and crack front location, the classic approach (e.g. hypothesizing a SCDFM and then comparing it against experimental evidence) may not be adequate. To overcome this limitation and perform a data driven correlation analysis, we chose to employ a $\mathrm{BN}$ approach due to its unique features:

- Being a probabilistic framework, the uncertainty of BN predictions can be quantified.

- Hidden variables can be discovered through data analysis.

- The so-called 'prior knowledge' can be modeled and tested against the data very easily.

- Thanks to graph theory, BN can be represented as direct graph allowing for a clear interpretation of the results, compared to others machine-learning frameworks (such as neural network).

- Being a non-parametric framework, no equation is required, in order to establish a relationship between different variables.

Based on the aforementioned benefits, BNs are becoming an increasingly popular tool within the scientific community. Just to point out one of the relevant example in this field, Sanakararaman et al [51] utilized BNs to quantify and validate fatigue crack growth predictions. Furthermore, the software package utilized in this work to create, manipulate, and query BNs is BayesiaLab v6.0.2.

Once data have been collected through the mining procedure describe in the previous section, the following variables are selected for the analysis:

- The Euclidean distance from the closest point to the crack front $\left(D_{\mathrm{CF}}\right)$.

- The FIP value (FIP) (each FIP is analyzed separately).

- RL (for points which will not have an associated RL due to non-failure or infinite life, a specific state, referenced as ${ }^{*}$ will be used).

A crucial aspect of the $\mathrm{BN}$ is the discretization of the variables while retaining their correct distribution. A genetic algorithm has been utilized to choose the most appropriate 
number of bins together with their support width. A complete list of the discretization results is given in the appendix. Once the variables have been discretized, the network can be built. In this work, we use expert knowledge to construct the networks, e.g. relationship between the different variables are imposed. In order to understand whether a specific value of any FIPs is able to adequately describe the short crack behavior, a static BN has been constructed. This means that, even if a specific location is mined more than once, i.e. integration points with an associated RL, each observation is treated as independent. In other words, the evolution of the FIPs' value w.r.t. time (cycles) is not taken into account. It should be noted that network parameters, correlation analysis, and predictions will be performed utilizing the entire dataset resulting from the aforementioned mining procedure. As will be explained later, $K$-fold cross-validation will be utilized to ensure convergence of the results.

In order to test whether the amount of information carried by the aforementioned SCDFM is sufficient to correctly estimate the expected RL, the network will be built following the subsequent assumptions:

- $D_{\mathrm{CF}}$ is the root of the direct acyclic graph.

- FIP is a child of $D_{\mathrm{CF}}$.

- RL is a child of FIP and $D_{\mathrm{CF}}$.

Hence, the joint probability distribution of our network can be written as:

$$
P\left(\mathrm{FIP}, \mathrm{RL}, D_{\mathrm{CF}}\right)=P\left(\mathrm{RL} \mid \mathrm{FIP}, D_{\mathrm{CF}}\right) P\left(\mathrm{FIP} \mid D_{\mathrm{CF}}\right) P\left(D_{\mathrm{CF}}\right) \text {. }
$$

Once the dependence between the different variables has been established, conditional probability tables, which are required to compute the posterior distributions, can be constructed directly exploiting the frequency of the data and their discretization. When all network parameters have been computed, we can assess the performance of each of the eight networks incorporating the different FIP formulations.

Building distributions only from available data, in which the complete 'state of nature' may not be available, is always risky. Parameters of each network and their relative influence may be different and not accurate if applied to a different dataset. In this work, a $K$-fold cross validation method (i.e. randomly select $(100 / K)(K-1) \%$ of the sample dataset as a training set to build the conditional distribution tables, and use the remaining $(100 / K) \%$ as validation, and repeat $K$ times, in this study $K=10$ ) has been applied to ensure that networks parameters are independent from the subset of data utilized to compute them. Results of the $K$-fold validation are in agreement with the BNs utilized to present the results.

Performance capability of a model can be evaluated utilizing reliability and precision. Recalling that the variables have been discretized, reliability (equation (14)) and precision (equation (15)) for each bin is defined respectively as:

$$
\begin{aligned}
& \text { Reliability }_{i, j}=\frac{\# \text { Predicions }_{i, j}}{\text { Total \# Prediction }_{i}}, \\
& \text { Precision }_{i, j}=\frac{\# \text { Predicions }_{i, j}}{{\text { Total } \# \text { Observations }_{j}}},
\end{aligned}
$$

where the index $i, j$ identify the index associated with the bin of predictions and observations respectively. Moreover, a mean reliability and precision (equations (16) and (17)) can also be defined as

$$
\overline{\text { Reliability }}=\frac{1}{\text { NBins }} \sum_{i=1}^{\text {NBins }} \text { Reliability }_{i, i},
$$




$$
\overline{\text { Precision }}=\frac{1}{\text { NBins }} \sum_{i=1}^{\text {Nsins }} \text { Precision }_{i, i},
$$

where $\delta_{i, j}$ is the Kronecker delta operator. To elucidate the role played by each FIP's formulation in predicting the observed RL, three different metrics are used:

1. Pearson's correlation coefficient (PCC) [52]:

$$
\rho_{X, Y}=\frac{\operatorname{cov}(X, Y)}{\sigma_{X} \sigma_{Y}}
$$

where $X, Y$ are random variables and $\sigma_{X}, \sigma_{Y}$ represent the standard deviation of $X, Y$, respectively. This is the most commonly used correlation metric, and it is only capable of correctly identifying linear correlations. This means that if a correlation metric is used for badly scaled data (e.g. Paris law not in the $\log$ space), the correlation coefficient $\rho$ will over or underestimate the relationship. Another aspect to consider is that if the correlation is almost linear, this metric is able to identify the sign of the correlation.

2. Mutual information (MI) [53] and symmetric normalized mutual information (SNMI):

$$
I(X, Y)=\sum_{x \in X} \sum_{y \in Y} p(x, y) \log _{2} \frac{p(x, y)}{p(x) p(y)}
$$

where $x, y$ are respectively a specific state of $X, Y, p(x), p(y)$ are the marginal distributions of $X, Y$, respectively and $p(x, y)$ is the joint probability of $X, Y$. MI is a measure of the amount of information shared by the random variables $X$ and $Y$. In other words, it measures how much knowing one variable reduces the uncertainty about the other. In contrast to PCC, MI contains information of both linear and nonlinear dependencies making it a more appropriate measure for deterministic correlations.

A limitation of MI is that it does not have an upper bound as is the case for the PCC. In order to have a more meaningful value, MI can be normalized utilizing the entropies of its random variables. There are many possibilities to normalize MI. The so called SMMI has been adopted, and is defined by the following:

$$
\operatorname{SNMI}(X, Y)=2 \frac{I(X, Y)}{H(X)+H(Y)},
$$

where $H(X)=-\sum_{x \in X} p(x) \log _{2}(p(x))$ is the entropy of the random variable $X$.

3. Kullback-Leibler divergence $\left(D_{\mathrm{KL}}\right)[54]$ :

In some situation, $\mathrm{MI}$ is not able to capture relationship even if they exist (e.g. XOR ${ }^{9}$ operator). Hence, the Kullback-Leibler divergence, $D_{\mathrm{KL}}$, has been adopted as a third metric to double check results of PCC and MI. $D_{\mathrm{KL}}$ is defined as follows:

$$
D_{\mathrm{KL}}(P(X) \| Q(X))=\sum_{x \in X} P(x) \log _{2} \frac{P(x)}{Q(x)},
$$

where $P$ and $Q$ are two different probability distributions, respectively representing the real and surrogate distributions. For what will follow, $P$ is determined using equation (13), while $Q$ is an approximation of $P$, where no link between the variables FIP and RL exists, hence:

$$
Q\left(\mathrm{FIP}, \mathrm{RL}, D_{\mathrm{CF}}\right)=P\left(\mathrm{RL} \mid D_{\mathrm{CF}}\right) P\left(\mathrm{FIP} \mid \mathrm{D}_{\mathrm{CF}}\right) P\left(D_{\mathrm{CF}}\right)
$$

$D_{\mathrm{KL}}$ represents the amount of information lost utilizing $Q$ to approximate $P$. In contrast to MI, $D_{\mathrm{KL}}$ is not limited in comparing the distribution of two random variables, but can be used

9 Exclusive OR operator. 
to directly compare two joint distribution. In other words, $D_{\mathrm{KL}}$ is able to capture existing correlations requiring the identification of more than two variables. As a note, while the PCC ranges between -1 and $1, \mathrm{MI}$ and $D_{\mathrm{KL}}$ start from 0 and have no upper bounds, thus the results are meaningful only as a comparison. Moreover, $\mathrm{MI}$ and $D_{\mathrm{KL}}$ are more resilient than PCC to the presence of outliers [55].

As with every model, BNs have limitations. Jensen et al [56] proposed a conflict metric to check if the BN model is well suited to predict a certain scenario. The idea is that predictions originating from a plausible set of evidence should exhibit a higher probability than considering a model in which all the variables are conditionally independent (i.e. the so called straw model). This conflict metric is formally defined as follow:

$$
\mathrm{GC}(E)=\log _{2}\left(\frac{\prod_{k=1}^{n} P\left(e_{k} \mid e_{k-1}, \ldots, e_{1}\right)}{\prod_{k=1}^{n} P\left(e_{k}\right)}\right),
$$

where, $E$ is a vector representing a set of evidences of length $k$ and $e_{k}$ is $k$ th evidence. The denominator is the product of the marginal distribution of each piece of evidence, and the numerator is the product of the conditional distribution of each evidence after all previous evidence has been instantiated. In other words, the numerator is the probability to observe a certain set of evidence given the BNs' model.

If the result in equation (23) is positive (i.e. $\mathrm{GC}(E)>0$ ), the evidence is supporting the model, which means that the model is well suited to predict the specified scenario. In contrast, if $\mathrm{GC}(E)<0$, then two possibilities arise: (i) the instantieated evidences are in conflict with the model, meaning that evidences represent a scenario not supported by the model, or (ii) a rare event has been identified. Unfortunatly, it is not possible to discern between the two aforementioned possibilities just looking at the $\mathrm{GC}(E)$ value, but it is still possible to identify the type of conflict through causal reasoning. This conflict metric will be utilized to elucidate some of the BNs prediction results.

\section{Results and discussion}

\subsection{Simulation results}

After the simulations are completed, the values for the FIPs are calculated based on the micromechanical fields (see equations (5)-(12)) at each spatial location, for each simulation. For example, figure 7 depicts the FIP values projected on the crack surface at cycle 53100 .

It should be noted that the color scale for slip-based FIPs (i.e. D1, D2, D3, D5) is different from the one utilized for energy-based FIPs (i.e. E1, E2, E3, E5). Moreover, the color scale of energy-based FIPs is shifted by two orders of magnitude to account for the scaling effect given by the resolved shear stress, which is on the order of hundreds of MPa. The gray region represents the area of the crack surface which has already undergone failure.

The first thing that can be noticed is that, except for $D 3 / E 3$, results appear to be comparable for slip/energy based FIPs, respectively. Focusing on slip based FIPs, which are representative of the plastic activity, only a small region ahead of the crack front exhibits relevant plasticity. It should also be recalled that these plots only represent values on the surface of the final crack volume (e.g. the crack surface is voxelized), and that higher plastic activity is present inside the volume. Another feature of the FIPs is their spatial heterogeneity, which could span orders of magnitude within a small spatial region adjacent to a GB (e.g. see figure 7 box 1). Moreover, locations far away from the crack front where damage starts to build up can be identified (i.e. see box 2 in figure 7). These aforementioned regions could 


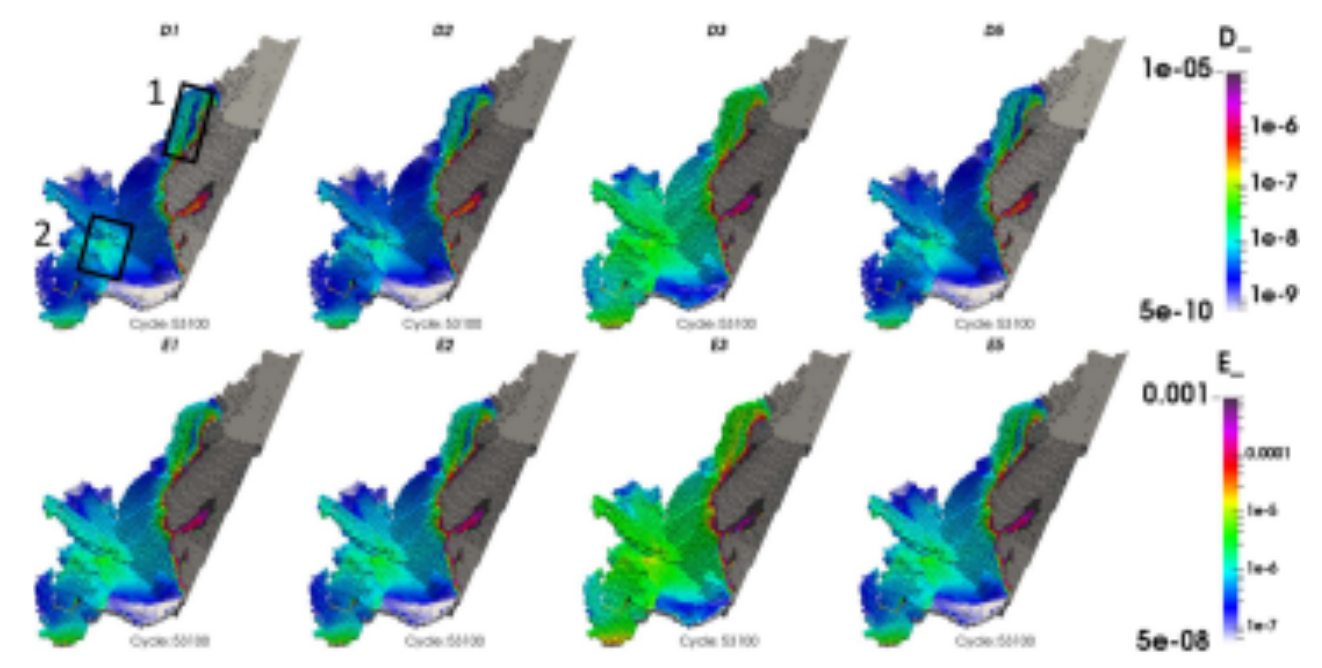

Figure 7. Comparison of the eight different FIPs formulations projected on the crack surface at cycle 53100 . The gray region represents the area of the crack which has already failed.

possibly identify likely failure locations as the crack continues to propagate. The consistency in behavior between $D 1$ and $D 2$ is not surprising, given that the former represents the slip system, and latter represents the slip plane with the maximum accumulated plastic shear. In fact, recalling that the material is BCC and that all 48 the slip systems have been utilized in the simulations, it is very likely to find that the plane with maximum accumulated plastic shear has only one slip system. In contrast, the similarity between D2 and D5 is more surprising.

The accumulated shear strains across all slip systems, D3, is the only one of the slip based FIPs showing a different trend. Upon further analysis, the location of areas exhibiting high/low values remains stable and the difference is only a shift on the color scale towards higher values. The same considerations can be applied to the energy-based FIPs. Moreover, comparing the spatial distribution between slip- and energy-based FIPs, it can be notice that the trend is the same except for a scaling factor (see color bar scale).

The material within this study exhibits work softening behavior and the fatigue experiment is loaded with $R_{\sigma}=0.0325$ (see table 1), it may be expected that once a slip system is activated, due to its orientation and loading condition a sort of avalanche mechanism will manifest through cycling. In other words, the most active slip system will remain stable between one cycle and the next. As a consequence, a major difference is not expected, between the most active system $(D 1)$, the most active plane $(D 2)$, or the total accumulate slip (D3). These considerations explain the similarity of the behavior observed utilizing $D 1, D 2$, and $D 3$, which are based only on the accumulated slip. Moreover, the aforementioned avalanche mechanism will persist until the crack geometry is updated and the overall load is redistributed.

For the similarity between $D 5$ and the other slip-based FIPs, it should be recalled that during the HCF regime, the plastic zone ahead of the crack front is small. Moreover, due to the formulation of D5 (see equation (12)), the opening stress plays a role only if plastic activity is present. The combination of the aforementioned observations elucidate why D5 is equivalent to other FIPs. 


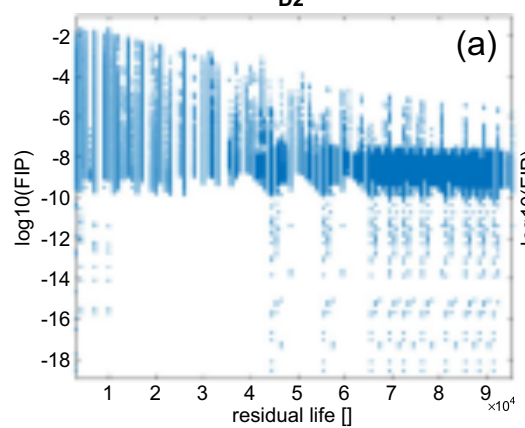

D2

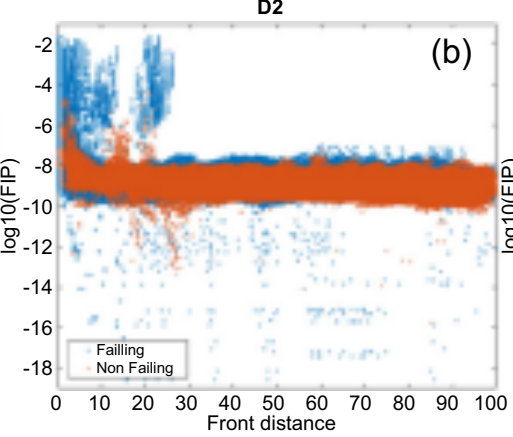

PDF

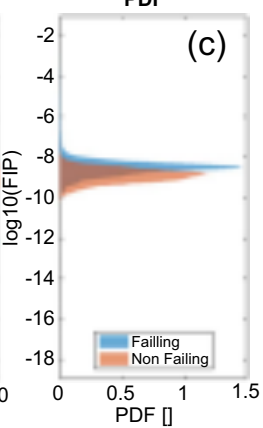

Figure 8. (a) Scatter plot of the $\log$ of $D 2$ values against observed residual life; (b) scatter plot of the $\log$ of $D 2$ values against the distance from the crack front; (c) marginal distribution of the $D 2$ values for failing and non-failing data.

To explain the similarity between the slip and energy-based FIPs, we need to look back at the flow rule (see equation (1)). Analyzing the equation, it can be noticed that an exponential relationship between the applied resolved shear stress and slip rate exists, thus explaining the equivalence between the slip and energy based FIPs. Further, due to the very similar behavior of all the FIPs, $D 2$ has been selected as a representative candidate, and several of the results that will follow will be presented only for the $D 2$ FIP. However, the same analysis has been performed for all the investigated FIP formulations, leading to the same conclusions.

\subsection{Data mining results}

Once all FIPs have been computed, the mining procedure described earlier was used to gather the required data. Figure 8(a) is a scatter plot representing the joint distributions of the computed $D 2$ values and the observed RL (i.e. $P($ FIP, RL)). Similarly, figure $8(\mathrm{~b})$ shows the joint distributions of $D 2$ values and the distance from the crack front (i.e. $P\left(\mathrm{FIP}, D_{\mathrm{CF}}\right)$ ). The blue color is associated to data representing failure, while the orange one represents data associated with non-failing behavior. Moreover, it should be noted that because only failing locations have an associated RL values, only these values can be correctly shown in figure 8(a); data coming from non-failing locations would appear as a vertical line at infinity. Figure 8(c) represent the conditional distributions of $D 2$ for the failing points, namely $P(\mathrm{FIP} \mid$ Failing), and of the non-failing points, $P(\mathrm{FIP} \mid$ NonFailing $)$. The marginal distribution of the FIP can be computed marginalizing the two aforementioned conditionals (i.e. $P($ FIP $)=P($ FIP $\mid$ Failing $)+P($ FIP $\mid$ NonFailing $)$. As a note, all the vertical axes have the same scale.

Focusing on figure $8(a)$, the first noticeable feature is how the data clusters in vertical bands. It should be noted that these bands form because only specific RL values are available from the experiments. Moreover, RL ranges approximately from 1000 to 100000 cycles. Focusing on the scale of the $D 2$ value, it can be noticed that only few data points exhibit considerable plastic activity, meaning that the plastic zone ahead of the crack tip is very limited, which is typical of the HCF regime. Additionally, a clear pattern can be identified. Moving from low to high RL (i.e. from left to right), the spread in the data decreases, together with their average magnitude values, indicating that no plastic activity is present. The $D 2$ values representing non-failing behavior can be correctly positioned in figure $8(\mathrm{~b})$. As a general behavior, it can be noticed that when the distance from the crack front (i.e. $D_{\mathrm{CF}}$ ) 
Table 4. Mean reliability and precision of the different BNs.

\begin{tabular}{|c|c|c|}
\hline & $\begin{array}{l}\text { Mean } \\
\text { reliability }\end{array}$ & $\begin{array}{c}\text { Mean } \\
\text { Precision }\end{array}$ \\
\hline$D 1$ & $38 \%$ & $30 \%$ \\
\hline$D 2$ & $41 \%$ & $31 \%$ \\
\hline D3 & $33 \%$ & $27 \%$ \\
\hline$D 5$ & $41 \%$ & $31 \%$ \\
\hline$E 1$ & $36 \%$ & $31 \%$ \\
\hline$E 2$ & $36 \%$ & $31 \%$ \\
\hline$E 3$ & $34 \%$ & $28 \%$ \\
\hline$E 5$ & $40 \%$ & $31 \%$ \\
\hline
\end{tabular}

exceed $42 \mu \mathrm{m}$ (i.e. 30 voxels or two-thirds of the average grain size), the data coming from the failing/non-failing locations overlap. In contrast, for $D_{\mathrm{CF}}<42 \mu \mathrm{m}$, a major overlap still exists, but a considerable number of points exhibit a much higher $D 2$ value. This confirms our hypothesis that plasticity is not the only key factor driving the crack propagation rate.

Focusing on the conditional distributions shown in figure 8(c), it can be noticed that the modal values of failing points is higher than the one observed for the non-failing ones. Another difference between the aforementioned distributions is that the one representing failure exhibits a much longer tail towards high FIP values than the other one. These are indications that, in general, failure can be related to relevant plastic activity, but their correlation appears to be weak.

\subsection{BNs results}

After the BNs have been defined and distributions discretized, we can instantiate evidence and compute inferences. This means that we can utilize data mined from the simulations and the experiment to query the networks and compute the predicted RL. Moreover, because the $\mathrm{BN}$ results are in terms of posterior probability (PP) of each possible outcome, we selected the outcome exhibiting the maximum likelihood as the correct prediction. To evaluate average performance of the different BN's, their mean reliability and precision have been computed (see equations (16) and (17)). Results are presented in table 4.

From table 4 , it is observed that all the BNs exhibit the same behavior with very small fluctuations. Reliability ranges from $33 \%$ to $41 \%$, while precision ranges from $27 \%$ to $31 \%$. Hence the different networks can be considered equivalent, which is not surprising given the similarity in behavior between all the FIPs. It is noted that the performance of D3 and E3 is slightly worse than the other FIPs. This means that total plasticity could be considered as a worse indicator of crack propagation between the selected FIPs.

In order to quantify the correlation between each FIP and the RL, PCC, MI, $D_{\mathrm{KL}}$, and SNMI have been computed, and are presented in table 5. It should be recalled that these metrics are computed utilizing marginal distributions of the variables and should be interpreted in an average sense. In other words, the correlation could be weak, if the overall distribution is considered; but on the other hand, the correlation could be stronger, if the analysis is restricted to a specific range. The first thing noticed analyzing the values of the PCC, is that an inverse correlation exists between all the FIPs and RL. In other words, as the FIPs value increase, RL decreases. This behavior is expected and can be easily interpreted: as damage continues to accumulate, the likelihood of failure increases. Moreover, all the FIPs 
Table 5. Correlations between the discretized FIPs distribution and the residual life distribution. Longer bars represent higher correlation. Note that while Pearson correlation is able to capture the sign of the correlation, mutual information and KullbackLeibler divergence are always positive by definition (equations (18)-(21)).

\begin{tabular}{|c|c|c|c|c|}
\hline & \multicolumn{4}{|c|}{ Correaltions metric: FIPs vensus RL } \\
\hline & PCC & MI & $D_{\mathrm{KL} .}$ & SNMI \\
\hline$D 1$ & $-24 \%$ & 0.041 & 0.062 & $3 \%$ \\
\hline$D 2$ & $-24 \%$ & 0.043 & 0.078 & $3 \%$ \\
\hline D3 & $-25 \%$ & 0.041 & 0.074 & $3 \%$ \\
\hline D5 & $-25 \%$ & 0.043 & 0.077 & $3 \%$ \\
\hline$E 1$ & $-27 \%$ & 0.042 & 0.060 & $3 \%$ \\
\hline$E 2$ & $-27 \%$ & 0.041 & 0.059 & $3 \%$ \\
\hline$E 3$ & $-28 \%$ & 0.039 & 0.054 & $3 \%$ \\
\hline E5 & $-28 \%$ & 0.050 & 0.082 & $3 \%$ \\
\hline
\end{tabular}

exhibits almost the same PCC, which is $\sim 26 \%$. To cross-check the linearity of the correlations and the equivalence between all the FIPs and RL, MI and $D_{\mathrm{KL}}$ have been computed. As can be seen in table 5 , the MI and $D_{\mathrm{KL}}$ results are in agreement with the PCC results.

Moreover, to quantify the amount of uncertainty reduction based on the RL by knowing the FIP values, the SNMI have been computed. As can be seen in table 5, the uncertainty reduction provided by FIPs is around 3\%. This means that only a small amount of information regarding failure is present in the FIPs distributions. It should also be mentioned that SNMI have been computed between RL and $D_{\mathrm{CF}}$ and it is approximately $14 \%$. This means that the distance from the crack front is more related to the observed RL than any of the computed FIPs.

Mean prediction results and their associated correlations provide valuable information, but to elucidate the ability of the FIPs to predict RL, BNs inference need to be analyzed in more detail. The analysis continues comparing the BNs inferences for each RL regime (i.e. each bin resulting from the discretization of the RL marginal distribution). This is necessary to reveal if stronger correlations exist for any specific RL regime. In fact, given the analysis of the joint and conditional distributions already performed, better predictions of the material behavior should be expected just ahead of the crack front. Complete reliability and precision results (see equations (14) and (15), respectively) of the BNs built upon the aforementioned D2 FIP values are presented in table 6.

Table 6 represents the quality of the predictions obtained by the BN based upon the D2 FIP. For each table: (i) columns represent experimental RL; (ii) rows represent the BN inferences; (iii) all rows and column headers describe each bins' support and the values in parenthesis represent the total number of observations/predictions of each bin; (iv) the last row and the last column, which are identified by the * represent the non-failing behavior; (v) color coding obeys the following rules: (a) blue highlights correct predictions in agreement with experimental observations (i.e. the prediction falls in the correct bin); (b) red highlights incorrect predictions; (c) color intensity represents the normalized amount of predictions falling in each bin (i.e. a deeper/lighter color means a higher/lower amount of prediction in the specific bins); (vi) the normalization constant utilized to compute percentages is: (1) the total number of predictions falling in each bin for reliability (i.e. rows), and (2) the total number of 
Table 6. Prediction quality of the network built upon $D 2$ : (a) reliability results (see equation (14)) and (b) precision results (see equation (15)). Rows represents predictions, and column represents experimental observations. Element on the main diagonal of each table (colored in blue) represent correct prediction, while element off of the main diagonal represents incorrect predictions. Green lines represent the RL limit for trustable predictions, purple lines identify the region belonging to the non-failing behavior.

\begin{tabular}{|c|c|c|c|c|c|c|c|c|c|c|c|c|c|c|c|c|c|}
\hline \multicolumn{9}{|c|}{ Reliability } & \multicolumn{9}{|c|}{ Precision } \\
\hline (a) & 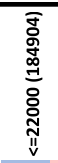 & 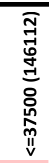 & 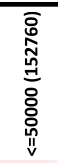 & 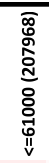 & 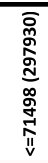 & 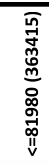 & 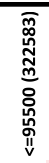 & 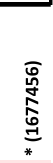 & (b) & 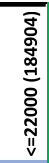 & 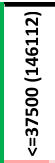 & 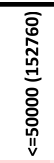 & 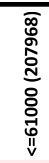 & 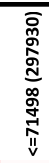 & 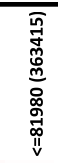 & 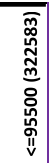 & 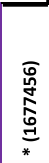 \\
\hline$<=22000(225275)$ & $48 \%$ & $22 \%$ & $9 \%$ & $7 \%$ & $2 \%$ & $1 \%$ & $0 \%$ & $10 \%$ & $<=22000(225275)$ & $59 \%$ & $34 \%$ & $14 \%$ & $8 \%$ & $2 \%$ & $1 \%$ & $0 \%$ & $1 \%$ \\
\hline$<=37500(145832)$ & $23 \%$ & $23 \%$ & $7 \%$ & $9 \%$ & $12 \%$ & $4 \%$ & $1 \%$ & $22 \%$ & $<=37500(145832)$ & $18 \%$ & $23 \%$ & $7 \%$ & $7 \%$ & $6 \%$ & $1 \%$ & $0 \%$ & $2 \%$ \\
\hline$<=50000(15246)$ & $8 \%$ & $23 \%$ & $42 \%$ & $13 \%$ & $1 \%$ & $0 \%$ & $0 \%$ & $13 \%$ & $<=50000(15246)$ & $1 \%$ & $2 \%$ & $4 \%$ & $1 \%$ & $0 \%$ & $0 \%$ & $0 \%$ & $0 \%$ \\
\hline$<=61000(166886)$ & $2 \%$ & $11 \%$ & $22 \%$ & $29 \%$ & $15 \%$ & $2 \%$ & $0 \%$ & $19 \%$ & $<=61000(166886)$ & $2 \%$ & $12 \%$ & $24 \%$ & $23 \%$ & $8 \%$ & $1 \%$ & $0 \%$ & $2 \%$ \\
\hline$<=71498(261506)$ & $0 \%$ & $0 \%$ & $9 \%$ & $21 \%$ & $30 \%$ & $24 \%$ & $4 \%$ & $11 \%$ & $<=71498(261506)$ & $0 \%$ & $0 \%$ & $15 \%$ & $27 \%$ & $26 \%$ & $18 \%$ & $3 \%$ & $2 \%$ \\
\hline$<=81980(268604)$ & $0 \%$ & $0 \%$ & $6 \%$ & $7 \%$ & $26 \%$ & $30 \%$ & $19 \%$ & $12 \%$ & $<=81980(268604)$ & $0 \%$ & $0 \%$ & $11 \%$ & $9 \%$ & $23 \%$ & $22 \%$ & $15 \%$ & $2 \%$ \\
\hline$<=95500(9250)$ & $0 \%$ & $0 \%$ & $0 \%$ & $0 \%$ & $0 \%$ & $16 \%$ & $58 \%$ & $25 \%$ & $<=95500(9250)$ & $0 \%$ & $0 \%$ & $0 \%$ & $0 \%$ & $0 \%$ & $0 \%$ & $2 \%$ & $0 \%$ \\
\hline${ }^{*}(2260529)$ & $2 \%$ & $2 \%$ & $2 \%$ & $2 \%$ & $5 \%$ & $9 \%$ & $11 \%$ & $67 \%$ & ${ }^{*}(2260529)$ & $21 \%$ & $28 \%$ & $26 \%$ & $25 \%$ & $35 \%$ & $57 \%$ & $79 \%$ & $91 \%$ \\
\hline
\end{tabular}

Table 7. Conflict analysis. (i) Column two and three represent the instantiated evidence (distance to the crack front and residual life, respectively), and (ii) column four represents the compute conflict metric, which if negative identify a possible conflict/ rare event.

\begin{tabular}{c|c|c|c}
\hline Scenario & $D_{\mathrm{CF}}$ & $\mathrm{RL}$ & $\mathrm{GC}(E)$ \\
\hline 1 & $<16$ & $<22 \mathrm{k}$ & 2.684 \\
\hline 2 & $<16$ & Non failing & -1.5729 \\
\hline 3 & $>84$ & $<22 \mathrm{k}$ & -6.2735 \\
\hline 4 & $>84$ & $82 \mathrm{k}<<95 \mathrm{k}$ & 0.495 \\
\hline
\end{tabular}

experimental observations in each bin for precision (i.e. columns). Also, the reliability/precision is computed by normalizing against the total number of predictions/observations for each bin, therefore the results of table 7 should be interpreted by rows/columns, respectively. Moreover, elements above the main diagonal represent predictions underestimating the RL, and element below the main diagonal represent predictions overestimating RL. The ideal case, in which all predictions are correct, is represented by diagonal matrices for both reliability and precision, if this is not the case an underestimation of the RL would represent a conservative engineering approach.

Analyzing the general appearance of the reliability table 6(a), it is noted that predictions are mainly positioned in a band around the main diagonal. This means that if a prediction is not correct, then it will under-/over-estimate RL in one of the adjacent bins. Analyzing prediction results from the experimental observation perspective (e.g. looking at the precision 

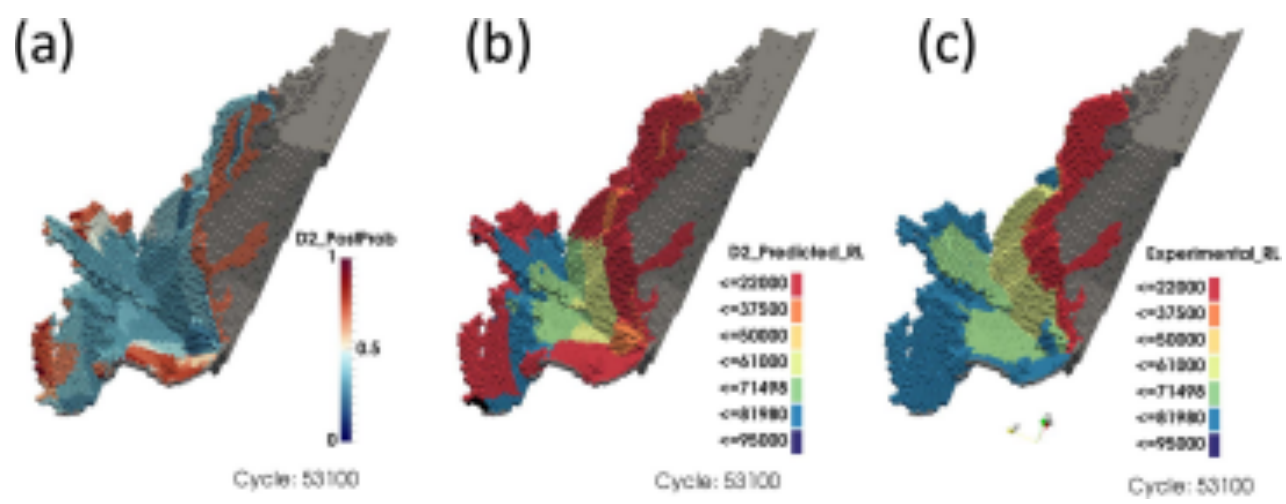

Figure 9. Comparison between BN results, and experimentally observed RL at cycle 51 100: (a) posterior probability of the most likely prediction, (b) predicted RL, and (c) experimentally observed RL.

table), the band behavior is still present, but some RL regime cannot be captured and is always over-/under-estimated (i.e. $37.5 \mathrm{k}<\mathrm{RL}<50 \mathrm{k}$ and $82 \mathrm{k}<\mathrm{RL}<95 \mathrm{k}$, columns 3 and 7 of table 6 (b) respectively). This is a first indication that, for some RL's regime, the model is not able to describe the material behavior.

Focusing on the diagonal elements (i.e. correct predictions) of the reliability table, we can notice that values ranges from $23 \%$ to $67 \%$. Specifically, more reliable predictions can be found close to the edge of the tables (i.e. first and last two rows). Moreover, the same tendency, but with higher fluctuation, is exhibited by reliability results. In other words, from a reliability and precision standpoint, good predictions are observed for very low or very high RL. In order for the model to be trustworthy, high values of both reliability and precision need to be present. Combining results of the reliability and precision and selecting only the bins exhibiting high values for both of them, only two regimes can be identified: (i) RL $<22 \mathrm{k}$, and (ii) non-failure regime. The former limit is visually identified in the tables by a green line, while the latter is identified by a purple line. Hence, all the RL regimes beyond $22 \mathrm{k}$ cycles (except for the non-failing behavior) should be considered as non-trustworthy. A special note should be added for predictions representing the non-failing data (i.e. the last row/column of the reliability/precision matrices, respectively, which are visually identified by purple lines). As can be seen in tables 6(a) and (b) the non-failing behavior appear to be the best captured behavior from both a reliability and precision standpoint.

Figure 9 depicts results obtained by the $\mathrm{BN}$ built upon $D 2$ compared to the experimentally observed RL projected on the crack surface at cycle 53100 . Specifically, subfigure (a) represents the PP values associate with the most likely predicted RL, (b) the predicted RL selected utilizing the maximum likelihood criteria, and (c) the experimentally observed RL. It should be noted, that color scale of the experimental RL has been discretized to ease comparison with predictions.

Comparing the predicted against the experimentally observed RL (figures 9(b), (c)), it can be noticed that there are regions in which predictions are in accordance with experimental observations, and regions in which the two differ. We start analyzing regions exhibiting prediction contradicting experimental observation. Some of these regions exhibit a nonconservative prediction of the RL. By investigating their associated PP values, it can be noticed that it is higher than $50 \%$, thus not a lot of uncertainty is associated to them. Moreover, if we identify their RL, we will notice that the aforementioned regions exhibit an 
experimental RL belonging to the non-trustworthy region thus making the prediction unreliable. The limit of the trustworthy region has been defined as a function of the observed RL, which is the unknown if one wants to use this model to predict failure. Hence, the obvious question is: 'How do we know which prediction should be trusted?' After analyzing all the predictions results, it has been found that in general, predictions belonging to the not-trustworthy regions behave as follows: (i) they are far away from the crack front; (ii) even if they exhibit a high PP, they are located beyond regions of very low PP; (iii) their computed RL would be lower than the one observed in adjacent regions closer to the crack front; and (iv) if trusted, they will generate a new crack disconnected from the main crack. Hence, they are easily identifiable and avoidable from a programming standpoint.

We now analyze predictions in accordance with experimental observations. As it can be noticed comparing figures 9(b) and (c), predictions in accordance with the experimental life are located close to the crack. Moreover, looking at the its PP, we also notice that only a very narrow band just ahead of the crack front exhibits a PP higher than 50\%. This behavior is expected, and is due to the very small plastic zone associated with HCF.

Instead of projecting the predictions on the crack surface and performing a visual qualitative analysis, a conflict analysis based upon the predictions results has been performed, in order to establish their trustworthiness. RL predictions have been performed imposing an observed FIP value and an observed $D_{\mathrm{CF}}$. For the conflict analysis, evidence will be imposed to ascertain if the data present in the FIP distribution will support the prediction. In other words, the expected RL and the $D_{\mathrm{CF}}$ will be instantiated and the conflict metric (see equation (23)) will be computed. Conflict analysis has been performed on the most relevant scenarios to determine if the prediction representing the imposed set of evidence is in conflict with the model, and the results are presented in table 7 .

The first column represents the scenario adopted in these analyses (see below), the second and the third columns represent instantiated evidence, and the fourth column represents $\mathrm{GC}(E)$ values computed according to equation (23). A blue bar means that the instantiated evidences are supporting the model, while a red bar means that a conflict or a rare event has been identified. Moreover, the longer the bar indicates a higher agreement/conflict.

Scenario 1 represents the failing behavior close to the crack front. As inferred by visual analysis of the predictions projected on the crack surface, this behavior is well captured by $\mathrm{BN}$, and the positive $\mathrm{GC}(E)$ value obtained for this scenario support our qualitative visual analysis that the FIPs are able to predict failure in the vicinity immediate ahead of the crack front.

Scenario 2 represents the non-failing behavior close to the crack front. As can be observed by the $\operatorname{GC}(E)$ value, the evidence is not supporting the model or a rare event is identified. In this case, it seems obvious that the non-failing behavior close to the crack front is not a rare event, in fact, looking at figure 4(b), the crack propagated only on the left side of the notch. Hence, it can be inferred that the model is not able to correctly capture this behavior.

Scenario 3 represents regions exhibiting low RL far away from the crack front. The $\mathrm{GC}(E)$ exhibit a highly negative value. This result further strengthens our qualitative analysis implying that predictions exhibiting this behavior should not be trusted.

Scenario 4 represents regions exhibiting high RL, far away from the crack front. In this case, the $\mathrm{GC}(E)$ value is slightly positive. The evidence is supporting the model but only marginally. As discussed before, far away from the crack, no significant plastic activity is observed, and hence the FIPs lose their ability to predict failure. However, partial information regarding failure are still embedded in the $D_{\mathrm{CF}}$ variable. The low support of the evidence is 
also shown by the low PP exhibited for predictions of regions belonging to this scenario (see figure 9(b)).

\section{Conclusion}

In this work, 3DXTSM results have been used as input for EVP-FFT CP simulations, in order to compute the micromechanical fields for each crack length within a HCF regime. The aforementioned results have been combined, and subsequently utilized to build BNs to test the ability of commonly used SCDFMs (FIPs) to predict SC growth. We have demonstrated that:

- Relationships embedded in the data are correctly captured as long as they exist.

- Predictions close to the crack front shows a satisfactory agreement with experimental observation.

- The PP associated with a prediction is a powerful tool to assess the prediction quality and the uncertainty associated with it.

- Utilizing conflict analysis, the range of applicability for the model can be easily identified, together with possible rare events.

The results of the $\mathrm{BN}$ demonstrate that the behavior of locally computed FIPs for the HCF regime display the following characteristics:

- All FIPs shows the same degree of correlation with respect to the experimentally observed RL.

- The effect of the opening stress on the critical plane does not bring additional information about the RL prediction, based on the current formulation.

- Due to the limited size of the plastic zone, FIPs show good predictions results only for a limited spatial and temporal range, i.e. close to the crack front and when observed RL is low.

- A possible avalanche mechanism has been hypothesized as the possible cause for the equivalent behavior exhibited by all the different FIPs in a softening material.

- The non-failing behavior close to the crack front, does not appear to be correctly captured by FIPs values.

- The distance from the crack front exhibits a stronger correlation with RL, than FIP values.

Results and interpretability of the established framework appear to be promising to establish the SCDF, and to identify a suitable expression for the SC growth rate, which could be used in finite elements computations to simulate the actual crack path. Moreover, further work is required in order to account for the non-local behavior of the phenomenon, to establish the role of other variables, and to include long-range effect due to inherent microstructure variability.

\section{Acknowledgments}

M D Sangid and A Rovinelli gratefully acknowledge support from the Air Force Office of Scientific Research under Contract No. FA9550-14-1-0284 (program managers J Fillerup and D Stargel). The authors would also like to thank Jean-Yves Buffière for his valuable insights and constructive discussions, Michael Herbig for his help during experiments, and Andrew King for important contributions to the development of the 3DXTSM technique. 

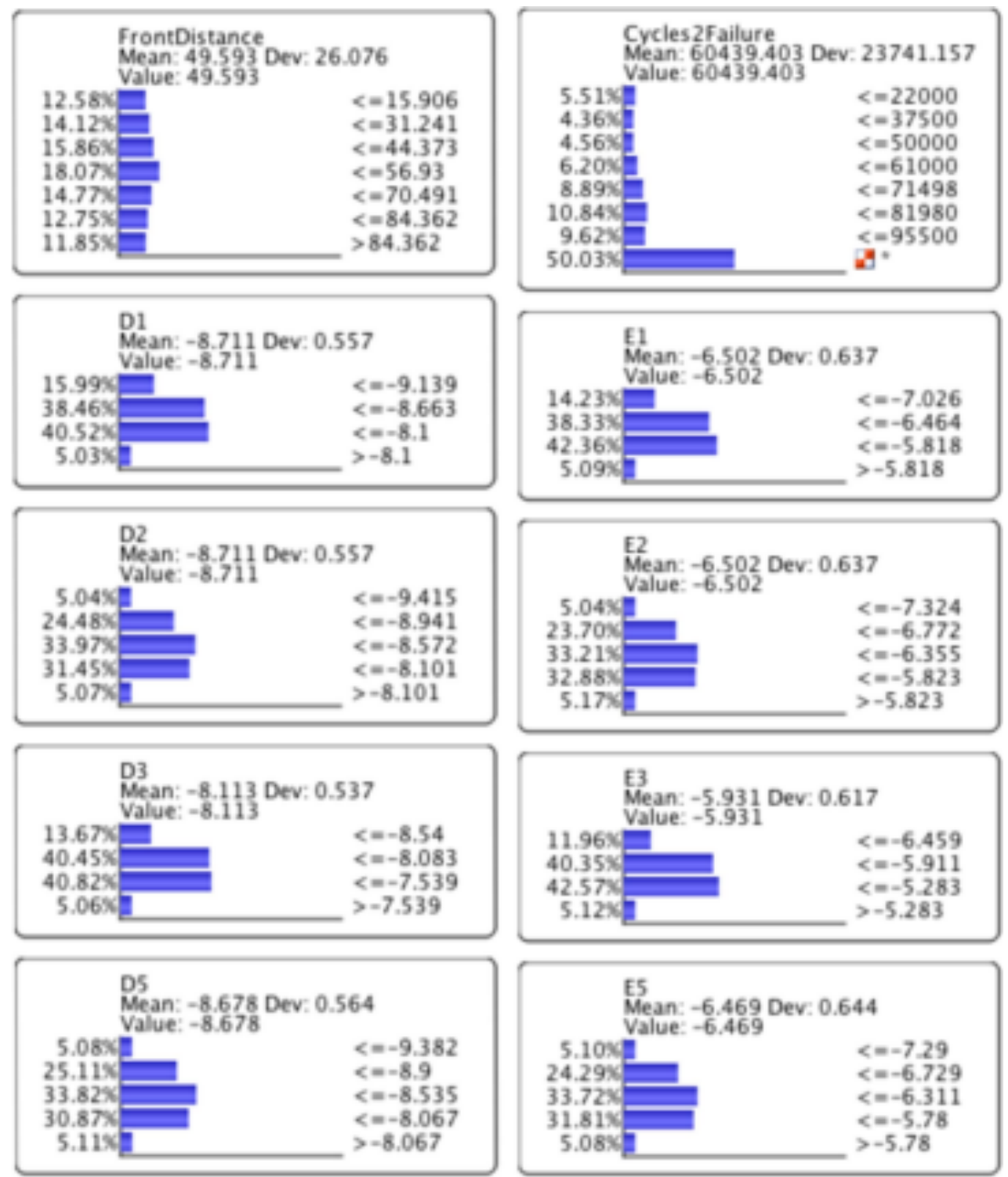

Figure A1. Discretization intervals, and marginal distributions of all the variables. It should be noted that FIP values intervals are logarithmic.

\section{Appendix}

\section{Discretization and marginal distributions}

Results of the discretization algorithm for all the variables utilized in this work. Intervals and resulting marginal distributions are depicted in figure A1. 


\section{References}

[1] Schijve J 2009 Fatigue of Structures and Materials 2nd edn (Dordrecht: Springer Netherlands) (https://doi.org/10.1007/978-1-4020-6808-9)

[2] Shiozawa K and Matsushita H 1996 Crack initiation and small fatigue crack growth behaviour of beta Ti-15V-3Cr-3Al-3Sn alloy Fatigue'96 Proc. 6th Int. Fatigue Congress p 301

[3] Paris P C and Erdogan F 1963 A critical analysis of crack propagation laws J. Basic Eng. (Trans ASME) 85 528-34

[4] Forsyth P J E 1961 A two stage process of fatigue crack growth Proc. Crack Propagation Symp. vol 1, pp 76-94

[5] Paris P C, Gomez M P and Anderson W E 1961 A rational analytic theory of fatigue Trend Eng. 13 9-14

[6] Pearson S 1975 Initiation of fatigue cracks in commercial aluminium alloys and the subsequent propagation of very short cracks Eng. Fract. Mech. 7 235-47

[7] Davidson D L, Chan K S, McClung R and Hudak S 2003 Small fatigue cracks Compr. Struct. Integr. 4 129-64

[8] Davidson D L, Chan K S and McClung R C 1996 Cu-bearing high-strength low-alloy steels: the influence of microstructure on the initiation and growth of small fatigue cracks Metall. Mater. Trans. A 27 2540-56

[9] Krupp U 2007 Fatigue Crack Propagation in Metals and Alloys: Microstructural Aspects and Modelling Concepts (New York: Wiley)

[10] Rovinelli A, Lebensohn R A and Sangid M D 2015 Influence of microstructure variability on short crack behavior through postulated micromechanical short crack driving force metrics Eng. Fract. Mech. 138 265-88

[11] Neumann P 1969 Coarse slip model of fatigue Acta Metall. 17 1219-25

[12] McEvily A J Jr and Boettner R C 1963 On fatigue crack propagation in f.c.c. metals Acta Metall. $11725-43$

[13] Rice J R and Thomson R 1974 Ductile versus brittle behaviour of crystals Phil. Mag. 29 73-97

[14] Asaro R J 1983 Crystal plasticity Trans. ASME, J. Appl. Mech. 50 921-34

[15] Wilkinson A J 2001 Modelling the effects of texture on the statistics of stage I fatigue crack growth Phil. Mag. A $81841-55$

[16] Ferrie E and Sauzay M 2009 Influence of local crystallographic orientation on short crack propagation in high cycle fatigue of 316 LN steel J. Nucl. Mater. 386-388 666-9

[17] Potirniche G P, Daniewicz S R and Newman J C 2004 Simulating small crack growth behaviour using crystal plasticity theory and finite element analysis Fatigue Fract. Eng. Mater. Struct. 27 59-71

[18] Bitzek E and Gumbsch P 2013 Mechanisms of dislocation multiplication at crack tips Acta Mater. 61 1394-403

[19] Sangid M D, Ezaz T, Sehitoglu H and Robertson I M 2011 Energy of slip transmission and nucleation at grain boundaries Acta Mater. 59 283-96

[20] Argon A S 2001 Mechanics and physics of brittle to ductile transitions in fracture Trans. ASME, J. Eng. Mater. Technol. 123 1-11

[21] Giannattasio A and Roberts S G 2007 Strain-rate dependence of the brittle-to-ductile transition temperature in tungsten Phil. Mag. 87 2589-98

[22] Guilhem Y, Basseville S, Curtit F, Stéphan J M and Cailletaud G 2010 Investigation of the effect of grain clusters on fatigue crack initiation in polycrystals Int. J. Fatigue 32 1748-63

[23] Sangid M D, Maier H J and Sehitoglu H 2011 An energy-based microstructure model to account for fatigue scatter in polycrystals J. Mech. Phys. Solids 59 595-609

[24] Mughrabi H 2009 Cyclic slip irreversibilities and the evolution of fatigue damage Metall. Mater. Trans. B 40 431-53

[25] Bennett V P and McDowell D L 2003 Polycrystal orientation distribution effects on microslip in high cycle fatigue Int. J. Fatigue $2527-39$

[26] Fatemi A and Socie D F 1988 Critical plane approach to multiaxial fatigue damage including outof-phase loading Fatigue Fract. Eng. Mater. Struct. 11 149-65

[27] Rice J R 1992 Dislocation nucleation from a crack tip: an analysis based on the Peierls concept J. Mech. Phys. Solids 40 239-71

[28] Tschopp M A and McDowell D L 2008 Influence of single crystal orientation on homogeneous dislocation nucleation under uniaxial loading J. Mech. Phys. Solids 56 1806-30 
[29] Hochhalter J D et al 2011 A geometric approach to modeling microstructurally small fatigue crack formation: III. Development of a semi-empirical model for nucleation Model Simul. Mater. Sci. Eng. 1935008

[30] Cerrone A et al 2015 Implementation and verification of a microstructure-based capability for modeling microcrack nucleation in LSHR at room temperature Model Simul. Mater. Sci. Eng. 2335006

[31] Yeratapally S R, Glavicic M G, Hardy M and Sangid M D 2016 Microstructure based fatigue life prediction framework for polycrystalline nickel-base superalloys with emphasis on the role played by twin boundaries in crack initiation Acta Mater. 107 152-67

[32] Castelluccio G M and McDowell D L 2015 Microstructure-sensitive small fatigue crack growth assessment: effect of strain ratio, multiaxial strain state, and geometric discontinuities Int. J. Fatigue 82 521-9

[33] Musinski W D and McDowell D L 2016 Simulating the effect of grain boundaries on microstructurally small fatigue crack growth from a focused ion beam notch through a threedimensional array of grains Acta Mater. 112 20-39

[34] Ludwig W et al 2009 New opportunities for 3D materials science of polycrystalline materials at the micrometre lengthscale by combined use of x-ray diffraction and x-ray imaging Mater. Sci. Eng. A 524 69-76

[35] Herbig M 2011 3D short fatigue crack investigation in beta titanium alloys using phase and diffraction contrast tomography PhD Thesis INSA-Lyon https://tel.archives-ouvertes.fr/tel00690521

[36] Herbig $\mathrm{M}$ et al 2011 3D growth of a short fatigue crack within a polycrystalline microstructure studied using combined diffraction and phase-contrast x-ray tomography Acta Mater. 59 590-601

[37] Proudhon H, Li J, Wang F, Roos A, Chiaruttini V and Forest S 2015 3D simulation of short fatigue crack propagation by finite element crystal plasticity and remeshing Int. J. Fatigue 82 $238-46$

[38] Li J, Proudhon H, Roos A, Chiaruttini V and Forest S 2014 Crystal plasticity finite element simulation of crack growth in single crystals Comput. Mater. Sci. 94 191-7

[39] Buffiere J-Y Y, Ferrie E, Proudhon H and Ludwig W 2006 Three-dimensional visualisation of fatigue cracks in metals using high resolution synchrotron x-ray micro-tomography Mater. Sci. Technol. 22 1019-24

[40] Vitek V 1968 Intrinsic stacking faults in body-centred cubic crystals Phil. Mag. 18 773-86

[41] Lebensohn R A, Kanjarla A K and Eisenlohr P 2012 An elasto-viscoplastic formulation based on fast fourier transforms for the prediction of micromechanical fields in polycrystalline materials Int. J. Plast. 32-33 59-69

[42] Moulinec H and Suquet P 1994 A fast numerical method for computing the linear and nonlinear mechanical properties of composites C. R. Acad. Des. Sci. II 318 1417-23

[43] Harren S, Lowe T C, Asaro R J and Needleman A 1989 Analysis of large-strain shear in ratedependent face-centred cubic polycrystals: correlation of micro- and macromechanics Phil. Trans. R. Soc. A 328 443-500

[44] Martin G 2012 Numerical multiscale simulation of the mechanical behavior of beta-metastable titanium alloys Ti5553 and Ti17 Thesis Ecole Nationale Supérieure des Mines de Paris https:// pastel.archives-ouvertes.fr/pastel-00873129

[45] Groeber M A and Jackson M A 2014 DREAM.3D: a digital representation environment for the analysis of microstructure in 3D Integr. Mater. Manuf. Innov. 3 1-17

[46] Rovinelli A, Proudhon H, Lebensohn R A and Sangid M D 2017 Assessing the reliability of FFTbased crystal plasticity simulations of a cracked polycrystalline material (in preparation)

[47] Fréour S, Lacoste E, François M and Guillén R 2011 Determining Ti-17 $\beta$-phase single-crystal elasticity constants through $\mathrm{x}$-ray diffraction and inverse scale transition model Mater. Sci. Forum 681 97-102

[48] Korsunsky A M, Dini D, Dunne F P E and Walsh M J 2007 Comparative assessment of dissipated energy and other fatigue criteria Int. J. Fatigue 29 1990-5

[49] Hochhalter J D et al 2010 A geometric approach to modeling microstructurally small fatigue crack formation: II. Physically based modeling of microstructure-dependent slip localization and actuation of the crack nucleation mechanism in AA 7075-T651 Model Simul. Mater. Sci. Eng. 18045004

[50] Dulmage A L and Mendelsohn N S 1958 Coverings of bipartite graphs Can. J. Math. 10 516-34 
[51] Sankararaman S, Ling Y and Mahadevan S 2011 Uncertainty quantification and model validation of fatigue crack growth prediction Eng. Fract. Mech. 78 1487-504

[52] Pearson K 1895 Note on regression and inheritance in the case of two parents Proc. R. Soc. London 58 240-2

[53] Shannon C E and Weaver W 1949 The Mathematical Theory of Information (Urbana, IL: University of Illnois Press)

[54] Kullback S and Leibler R A 1951 On information and sufficiency Ann. Math. Stat. 22 79-86

[55] Correa C D and Lindstrom P 2013 The mutual information diagram for uncertainty visualization Int. J. Uncertain. Quantif 3 187-201

[56] Jensen F V, Chamberlain B, Nordahl T and Jensen F 1990 Analysis in HUGIN of data conflict Proc. 6th Annual Conf. on Uncertainty in Artificial Intelligence pp 519-28 